TRANSACTIONS OF THE

AMERICAN MATHEMATICAL SOCIETY

Volume 359, Number 12, December 2007, Pages 5867-5898

S 0002-9947(07)04124-4

Article electronically published on July 20, 2007

\title{
GALOIS ACTIONS AND BLOCKS OF TAME INFINITESIMAL GROUP SCHEMES
}

\author{
ROLF FARNSTEINER AND ANDRZEJ SKOWROŃSKI
}

\begin{abstract}
Given an infinitesimal group $\mathcal{G}$, that is defined over an algebraically closed field of characteristic $p \geq 3$, we determine the block structure of the algebra of measures $H(\mathcal{G})$ in case its principal block $\mathcal{B}_{0}(\mathcal{G})$ is tame and the height of the factor group $\mathcal{G} / \mathcal{M}(\mathcal{G})$ of $\mathcal{G}$ by its multiplicative center $\mathcal{M}(\mathcal{G})$ is at least two. Our results yield a complete description of the stable Auslander-Reiten quiver of $H(\mathcal{G})$ along with a criterion for the domesticity of $H(\mathcal{G})$.
\end{abstract}

\section{INTRODUCTION}

One central question in the modern representation theory of algebras is the determination of the representation type. By Drozd's fundamental result (see [7, [10]) finite dimensional algebras over an algebraically closed field $k$ may be subdivided into three disjoint classes. For the class of representation-finite algebras, which have only finitely many isoclasses of indecomposable modules, the representation theory is well understood. The second class, called tame algebras, consists of representation-infinite algebras for which the indecomposable modules occur in each dimension in a finite number of discrete and a finite number of one-parameter families. The third class is formed by the wild algebras, whose representation theory comprises the representation theories of all finite dimensional algebras over $k$. Accordingly, a classification of the indecomposable finite dimensional modules is feasible only for representation-finite and tame algebras.

In continuation of [17] and [18] we investigate in this paper the representation theory of infinitesimal groups with tame principal blocks. Given such a group scheme $\mathcal{G}$, our object of study is the associated cocommutative Hopf algebra $H(\mathcal{G})$ (i.e., the algebra of measures on $\mathcal{G}$ ) and its principal block $\mathcal{B}_{0}(\mathcal{G})$. The results of [17] reveal major differences between this case and the well-understood module theory of finite groups: The absence of a suitable analogue for defect groups manifests itself in the somewhat surprising fact that an infinitesimal group with tame principal block may also possess some wild blocks. A precise criterion for the presence of this phenomenon was provided in [17] for infinitesimal groups of height $\leq 1$, and it is one objective of our present work to examine the situation for groups of height $\geq 2$.

Received by the editors November 15, 2004 and, in revised form, July 25, 2005.

2000 Mathematics Subject Classification. Primary 16G70; Secondary 14L15, 16G20, 16G60, 16W20, 16W30, 17B50.

This work was supported by Polish Scientific Grant KBN No. 1 PO3A 01827. 
Our extension of results on restricted Lie algebras to infinitesimal group schemes rests on an equivariant (or graded) version of the techniques employed in [17. The relevant results concerning graded modules of reduced enveloping algebras are recorded in Section 2. The next section shows how Galois extensions in the sense of 2] naturally appear in the context of infinitesimal group schemes. In fact, as we demonstrate in Theorem 3.7, the Hopf algebra extensions $H(\mathcal{G}): H(\mathcal{N})$ relevant for our purposes admit a free action by their Galois group, rendering the canonical restriction functor a Galois covering of module categories. In Section 4 we examine the block structures of the distribution algebras with tame principal blocks. The aforementioned Galois extensions afford a good control of the passage from $\mathcal{G}$ to a certain subgroup $\mathcal{N}$ that, in terms of its block theory, is closely related to the first Frobenius kernel $\mathcal{G}_{1}$. As a result, the occurrence of wild blocks turns out to be essentially a phenomenon of groups of height 1 - in all other cases the principal block is the most complicated block of the Hopf algebra $H(\mathcal{G})$. In particular, we prove

Theorem. Let $\mathcal{G}$ be an infinitesimal group of characteristic $p \geq 3$ such that the principal block of its Hopf algebra $H(\mathcal{G})$ is tame. Suppose that the height of the factor group of $\mathcal{G}$ by its multiplicative center $\mathcal{M}(\mathcal{G})$ satisfies $\operatorname{ht}(\mathcal{G} / \mathcal{M}(\mathcal{G})) \geq 2$, and let $\mathcal{B} \subset H(\mathcal{G})$ be a block.

(1) If $\mathcal{B}$ possesses a simple module of dimension $\neq p$, then $\mathcal{B}$ is representationinfinite and special biserial.

(2) If $\mathcal{B}$ affords a simple module of dimension $p$, then $\mathcal{B}$ is a Nakayama algebra.

In fact, in either case the basic algebras are given explicitly in terms of generators and relations, the structure of the group $\mathcal{G} / \mathcal{M}(\mathcal{G})$ is determined (see Theorems 4.3 and 4.5), and the number of blocks of each type is provided (see Corollary 4.4). Representation-infinite special biserial algebras form a distinguished class of tame algebras 43. Important examples of such algebras are provided by blocks of group algebras with dihedral defect groups [11, as well as the algebras appearing in the Gel'fand-Ponomarev classification of the singular Harish-Chandra modules over the Lorentz group [25].

In the last three sections we turn to topics related to Auslander-Reiten theory. Using results on special biserial algebras [12, we obtain the classification of the connected components of the stable Auslander-Reiten quiver of $\mathcal{G}$ for the case covered by the above result. In this context, the number $r:=\mathrm{ht}(\mathcal{G} / \mathcal{M}(\mathcal{G}))$ turns out to be related to the rank $\ell$ of the exceptional tubes via $\ell=p^{r-1}$. Proposition 5.2 identifies $p^{r-1}$ as the upper bound of such ranks for any infinitesimal group with symmetric Hopf algebra.

Section 6 provides an easy criterion for $H(\mathcal{G})$ to belong to the particularly tractable class of domestic algebras, which, in the classical setting of finite groups, are just the algebras whose Sylow-2-subgroups are Klein four groups. In our context, the representation-infinite blocks of such algebras are Morita equivalent to trivial extensions of radical square zero hereditary algebras of type $\tilde{\mathbb{A}}$ and thus are 2-parametric.

The final section applies Auslander-Reiten theory to identify the infinitesimal groups of finite Krull-Gabriel dimension.

Our arguments will heavily draw from [18, whose most frequently used results we record for the reader's convenience. Given $n \geq 0$ and $r \geq 1$, we denote by $\mathcal{W}_{n}$ and $\mathcal{Q}_{[r]}:=\mathrm{SL}(2)_{1} T_{r}$ the group of Witt vectors of length $n$ and the product of the first 
Frobenius kernel of SL(2) with the $r$-th Frobenius kernel of its standard maximal torus $T$, respectively. The following result shows that the groups of interest are central extensions of the groups $\mathcal{Q}_{[r]}$.

Theorem 1.1 (18, (3.5)). Let $\mathcal{G}$ be an infinitesimal group of characteristic $\geq 3$ such that $\mathcal{B}_{0}(\mathcal{G})$ is tame. Then there exist $r \geq 1$ and $n \geq 0$ such that $\mathcal{G} / \operatorname{Cent}(\mathcal{G}) \cong$ $\mathcal{Q}_{[r]}$ and $\operatorname{Cent}(\mathcal{G}) \cong \mathcal{M}(\mathcal{G}) \times\left(\mathcal{W}_{n}\right)_{1}$.

Here $\mathcal{M}(\mathcal{G})$ refers to the diagonalizable (multiplicative) part of the abelian group scheme $\operatorname{Cent}(\mathcal{G})$.

While the first Frobenius kernel $\mathcal{G}_{1}$ of an infinitesimal group $\mathcal{G}$ (or equivalently its Lie algebra $\operatorname{Lie}(\mathcal{G}))$ usually does not determine the structure of $\mathcal{G}$, it does capture essential structural features in the tame case. Note that preservation of tameness under passage to Frobenius kernels, stated in the following result, is not the consequence of a general descent argument, as subgroups of tame infinitesimal groups may very well be wild (cf. [17, §6]).

Theorem 1.2 ([18, (5.3)). Let $\mathcal{G}$ be an infinitesimal group of characteristic $\geq 3$. Then the following statements are equivalent:

(1) The principal block $\mathcal{B}_{0}(\mathcal{G})$ is tame.

(2) $\mathcal{B}_{0}\left(\mathcal{G}_{1}\right)$ is tame and $\mathcal{G} / \mathcal{G}_{1}$ is multiplicative.

\section{Graded MOdules of REDUCED ENVELOPING ALGEBRAS}

Throughout this paper, $k$ denotes an algebraically closed field of characteristic $p>0$. Unless mentioned otherwise, all algebras and modules are assumed to be finite dimensional. Given an associative $k$-algebra $\Lambda$, we denote by $\bmod \Lambda$ the category of (finite dimensional) left $\Lambda$-modules. If $\Lambda$ is graded relative to an abelian group $A$, we let $\bmod \Lambda^{\text {gr }}$ be the category of $A$-graded $\Lambda$-modules and degree zero homomorphisms.

We shall be interested in the case where $\Lambda$ is a reduced enveloping algebra of a restricted Lie algebra or a certain factor algebra thereof. We briefly introduce this family of Frobenius algebras and refer the interested reader to [41] for further details on restricted Lie algebras and their enveloping algebras.

Let $(\mathfrak{g},[p])$ be a restricted Lie algebra over $k$ with universal enveloping algebra $U(\mathfrak{g})$. For a linear form $\chi \in \mathfrak{g}^{*}$, we let

$$
U_{\chi}(\mathfrak{g}):=U(\mathfrak{g}) /\left(\left\{x^{p}-x^{[p]}-\chi(x)^{p} 1 ; x \in \mathfrak{g}\right\}\right)
$$

be the $\chi$-reduced enveloping algebra of $\mathfrak{g}$. For arbitrary $\chi \in \mathfrak{g}^{*}$ the algebra $U_{\chi}(\mathfrak{g})$ is a Frobenius algebra of dimension $p^{\operatorname{dim}_{k} \mathfrak{g}}$, while the restricted enveloping algebra $U_{0}(\mathfrak{g})$ inherits the structure of a (cocommutative) Hopf algebra from $U(\mathfrak{g})$. As such it is isomorphic to the algebra of distributions of an infinitesimal group scheme of height 1 (cf. [9, (II, $\S 7,4.2)]$ ). We denote by $\bmod (\mathfrak{g}, \chi)$ the category of $U_{\chi}(\mathfrak{g})$-modules. The $U_{\chi}(\mathfrak{g})$-modules are often also referred to as $\mathfrak{g}$-modules with $p$-character $\chi$.

Let $\mathcal{T}$ be an algebraic $k$-group scheme. In case $\mathcal{T}$ is reduced we shall use the equivalence $\mathcal{T} \mapsto \mathcal{T}(k)$ and identify $\mathcal{T}$-modules with rational $\mathcal{T}(k)$-modules. Suppose that $\mathcal{T}$ acts on a restricted Lie algebra $(\mathfrak{g},[p])$ via automorphisms of restricted Lie algebras, and let $\chi \in\left(\mathfrak{g}^{*}\right)^{\mathcal{T}}$ be an invariant relative to the contragredient action. By definition of $U_{\chi}(\mathfrak{g})$, there is a unique action of $\mathcal{T}$ on $U_{\chi}(\mathfrak{g})$ by automorphisms extending the operation of $\mathcal{T}$ on $\mathfrak{g}$. 
The following notion is closely related to Jantzen's $u_{n}$-T-modules (see [28, (2.1)]).

Definition. Let $(\mathfrak{g},[p])$ be a restricted Lie algebra, $\mathcal{T}$ an algebraic group that operates on $(\mathfrak{g},[p])$ via automorphisms, and $\chi \in\left(\mathfrak{g}^{*}\right)^{\mathcal{T}}$ a linear form. A $k$-vector space $M$ is called a $\left(U_{\chi}(\mathfrak{g}), \mathcal{T}\right)$-module if

(a) $M$ is a $U_{\chi}(\mathfrak{g})$-module and a $\mathcal{T}$-module, and

(b) $t(u m)=(t . u) t m$ for all $t \in \mathcal{T}, u \in U_{\chi}(\mathfrak{g})$, and $m \in M$.

Strictly speaking, the identity in part (b) above should hold for any commutative $k$-algebra $R$ and elements $t \in \mathcal{T}(R), u \in U_{\chi}(\mathfrak{g}) \otimes_{k} R$, and $m \in M \otimes_{k} R$. We will often suppress algebras when $k$-spaces are interpreted as affine schemes.

We let $\operatorname{Dist}(\mathcal{T})$ be the algebra of distributions on $\mathcal{T}$ (cf. [29, (I.7.7)]). If $\mathcal{T}=$ $\operatorname{Spec}_{k}(\mathcal{O}(\mathcal{T}))$ is an infinitesimal group scheme with function algebra $\mathcal{O}(\mathcal{T})$, then $\operatorname{Dist}(\mathcal{T})$ coincides with the Hopf algebra $H(\mathcal{T}):=\mathcal{O}(\mathcal{T})^{*}$, the so-called algebra of measures on $\mathcal{T}$. Since the multiplication $U_{\chi}(\mathfrak{g}) \otimes_{k} U_{\chi}(\mathfrak{g}) \longrightarrow U_{\chi}(\mathfrak{g})$ and the embedding $k \hookrightarrow U_{\chi}(\mathfrak{g})$ defined by the unity are homomorphisms of $\mathcal{T}$-modules, they are also $\operatorname{Dist}(\mathcal{T})$-linear (cf. [29, (I.7.11)]). In other words, letting $\varepsilon: \operatorname{Dist}(\mathcal{T}) \longrightarrow k$ denote the co-unit of the Hopf algebra $\operatorname{Dist}(\mathcal{T})$, we have

$$
h \cdot(u v)=\sum_{(h)}\left(h_{(1)} \cdot u\right)\left(h_{(2)} \cdot v\right) \quad \text { and } \quad h .1=\varepsilon(h) 1
$$

for every $h \in \operatorname{Dist}(\mathcal{T}), u, v \in U_{\chi}(\mathfrak{g})$, rendering $U_{\chi}(\mathfrak{g})$ a $\operatorname{Dist}(\mathcal{T})$-module algebra. Accordingly, we consider the smash product

$$
U_{\chi}(\mathfrak{g}) \sharp \operatorname{Dist}(\mathcal{T}),
$$

whose underlying $k$-space is the tensor product of the two defining algebras and whose product is given by

$$
(u \sharp h)\left(v \sharp h^{\prime}\right)=\sum_{(h)} u\left(h_{(1)} \cdot v\right) \sharp h_{(2)} h^{\prime} \quad \forall u, v \in U_{\chi}(\mathfrak{g}), h, h^{\prime} \in \operatorname{Dist}(\mathcal{T}) .
$$

We refer to [32, Chap. 4] for further properties of smash products.

Each $\left(U_{\chi}(\mathfrak{g}), \mathcal{T}\right)$-module naturally has the structure of a $\left(U_{\chi}(\mathfrak{g}) \sharp \operatorname{Dist}(\mathcal{T})\right)$-module. Moreover, if $\mathcal{T}$ is an infinitesimal group scheme, then $\bmod U_{\chi}(\mathfrak{g}) \sharp \operatorname{Dist}(\mathcal{T})$ is precisely the category of $\left(U_{\chi}(\mathfrak{g}), \mathcal{T}\right)$-modules.

Given an algebraic group scheme $\mathcal{G}$, we denote by $X(\mathcal{G}):=\operatorname{Hom}\left(\mathcal{G}, \mu_{k}\right)$ the character group of $\mathcal{G}$. If $\mathcal{G}$ is reduced, then $X(\mathcal{G}) \cong \operatorname{Hom}\left(\mathcal{G}(k), k^{\times}\right)$. For finite algebraic groups $X(\mathcal{G})$ is isomorphic to the group of algebra homomorphisms $H(\mathcal{G}) \longrightarrow k$ from the algebra of measures $H(\mathcal{G})$ to $k$ (see also Section 3 ). If $(\mathfrak{g},[p])$ is a restricted Lie algebra, then we define $X(\mathfrak{g})$ to be the group of algebra homomorphisms $U_{0}(\mathfrak{g}) \longrightarrow k$. This group corresponds to the set of those linear forms $f \in \mathfrak{g}^{*}$ that annihilate the subspace $[\mathfrak{g}, \mathfrak{g}]$ and satisfy $f\left(x^{[p]}\right)=f(x)^{p}$ for all $x \in \mathfrak{g}$.

Let $\mathcal{T}$ be an algebraic $k$-group scheme. We say that $\mathcal{T}$ is multiplicative or diagonalizable if its function algebra $\mathcal{O}(\mathcal{T})$ coincides with the group algebra $k[X(\mathcal{T})]$ of the finitely generated abelian group $X(\mathcal{T})$ (see [29, (I.2.5)]). Such a group scheme is called a torus if $X(\mathcal{T})$ is free, so that tori are reduced group schemes.

Now let $\mathcal{T}$ be a multiplicative group, acting on a restricted Lie algebra $(\mathfrak{g},[p])$ via automorphisms. Then the weight space decomposition (cf. [29, (I.2.11)])

$$
\mathfrak{g}=\bigoplus_{\lambda \in X(\mathcal{T})} \mathfrak{g}_{\lambda}
$$


endows $\mathfrak{g}$ with the structure of an $X(\mathcal{T})$-graded restricted Lie algebra. In particular, we have $\mathfrak{g}_{\lambda}^{[p]} \subset \mathfrak{g}_{p \lambda}$ for every element $\lambda \in X(\mathcal{T})$. The universal enveloping algebra inherits this gradation from $\mathfrak{g}$, and if $\chi \in\left(\mathfrak{g}^{*}\right)^{\mathcal{T}}$ is a $\mathcal{T}$-invariant linear form, then $\chi$ annihilates $\sum_{\lambda \neq 0} \mathfrak{g}_{\lambda}$, so that $U(\mathfrak{g})\left\{x^{p}-x^{[p]}-\chi(x)^{p} 1 ; x \in \mathfrak{g}\right\}$ is a homogeneous ideal of $U(\mathfrak{g})$. Accordingly, $U_{\chi}(\mathfrak{g})$ inherits the $X(\mathcal{T})$-grading from $\mathfrak{g}$, and this grading is induced by the action of $\mathcal{T}$ on $U_{\chi}(\mathfrak{g})$. We shall denote by $\bmod (\mathfrak{g}, \chi)^{\text {gr }}$ the category of $X(\mathcal{T})$-graded $\mathfrak{g}$-modules with $p$-character $\chi$. As was noted in [30, (2.3)] the category of $\left(U_{\chi}(\mathfrak{g}), \mathcal{T}\right)$-modules coincides with $\bmod (\mathfrak{g}, \chi)^{\mathrm{gr}}$ in case $\mathcal{T}$ is a torus.

Lemma 2.1. Let $\mathcal{T}$ be a torus acting on a restricted Lie algebra $(\mathfrak{g},[p])$ via automorphisms and suppose that $\chi \in\left(\mathfrak{g}^{*}\right)^{\mathcal{T}}$ is a $\mathcal{T}$-invariant linear form. Then the following statements hold:

(1) Every principal indecomposable $U_{\chi}(\mathfrak{g})$-module has the structure of a $\left(U_{\chi}(\mathfrak{g})\right.$, $\mathcal{T})$-module .

(2) Every simple $U_{\chi}(\mathfrak{g})$-module has the structure of a $\left(U_{\chi}(\mathfrak{g}), \mathcal{T}\right)$-module.

(3) The category $\bmod (\mathfrak{g}, \chi, \mathcal{T})$ of $\left(U_{\chi}(\mathfrak{g}), \mathcal{T}\right)$-modules has enough projectives.

Proof. (1),(2) As noted before, the action of $\mathcal{T}$ on $\mathfrak{g}$ and $U_{\chi}(\mathfrak{g})$ induces gradings relative to the finitely generated free abelian group $X(\mathcal{T})$. Let $M$ be a principal indecomposable or simple $U_{\chi}(\mathfrak{g})$-module. Thanks to [26, (3.5)] (see also [30, (1.4)]) the module $M$ is $X(\mathcal{T})$-gradable. As observed in [30, (2.3)], this gradation corresponds to a $\mathcal{T}$-action on $M$, which renders $M$ a $\left(U_{\chi}(\mathfrak{g}), \mathcal{T}\right)$-module.

(3) Let $M$ be a $X(\mathcal{T})$-graded $U_{\chi}(\mathfrak{g})$-module. Thanks to [30, (1.5)] the tops of $M$ in $\bmod (\mathfrak{g}, \chi)$ and $\bmod (\mathfrak{g}, \chi)^{\mathrm{gr}}$ coincide. In view of [30, (1.6)] the semisimple module $\operatorname{Top}(M)$ possesses a projective cover $P \stackrel{\varphi}{\longrightarrow} \operatorname{Top}(M)$ in $\bmod (\mathfrak{g}, \chi)^{\mathrm{gr}}$, whose image under the forgetful functor $\bmod (\mathfrak{g}, \chi)^{\operatorname{gr}} \longrightarrow \bmod (\mathfrak{g}, \chi)$ is a projective cover in $\bmod (\mathfrak{g}, \chi)$. Letting $\pi: M \longrightarrow \operatorname{Top}(M)$ be the canonical projection, there exists a surjective homomorphism $\psi: P \longrightarrow M$ of $U_{\chi}(\mathfrak{g})$-modules such that $\pi \circ \psi=\varphi$. Since $\varphi$ and $\pi$ are homogeneous maps of degree zero, decomposing $\psi$ into its homogeneous parts yields $\varphi=\pi \circ \psi_{0}$, whence $M=\psi_{0}(M)+\operatorname{Rad}(M)$. Accordingly, $\psi_{0}$ is the desired surjection.

In the sequel we shall focus our attention on certain central extensions of restricted Lie algebras that naturally arise in the context of tame infinitesimal groups (cf. [17]). Let $(\mathfrak{g},[p])$ and $(\mathfrak{a},[p])$ be restricted Lie algebras, and assume $\mathfrak{a}$ to be abelian. Given a $p$-semilinear map $\psi: \mathfrak{g} \longrightarrow \mathfrak{a}$, we denote by $\mathfrak{g}_{\psi}$ the central extension of $\mathfrak{g}$ by $\mathfrak{a}$, whose underlying $k$-space is $\mathfrak{g} \oplus \mathfrak{a}$, and whose bracket and $p$-map are defined by

$[(x, a),(y, b)]=([x, y], 0) \quad$ and $\quad(x, a)^{[p]}=\left(x^{[p]}, \psi(x)+a^{[p]}\right) \quad \forall x, y \in \mathfrak{g}, a, b \in \mathfrak{a}$,

respectively. Thus, the canonical embedding $\mathfrak{g} \hookrightarrow \mathfrak{g}_{\psi}$ is a homomorphism of ordinary Lie algebras, yet usually not one of restricted Lie algebras. Our next result provides a $\mathcal{T}$-equivariant version of [17, (8.2)].

Lemma 2.2. Let $\mathcal{T}$ be an algebraic group, acting on $\mathfrak{g}$ via automorphisms. Suppose that $\psi: \mathfrak{g} \longrightarrow \mathfrak{a}$ is a p-semilinear map into an abelian restricted Lie algebra $\mathfrak{a}$ such that $\psi(t . x)=\psi(x) \forall x \in \mathfrak{g}, t \in \mathcal{T}$. Then the following statements hold:

(1) Setting t. $(x, a):=(t . x, a)$ for $t \in \mathcal{T}, x \in \mathfrak{g}$ and $a \in \mathfrak{a}$, we obtain an action of $\mathcal{T}$ on $\mathfrak{g}_{\psi}$ via automorphisms. 
(2) Let $\gamma \in X(\mathfrak{a})$ be a character, and define $\chi_{\gamma} \in \mathfrak{g}^{*}$ via $\chi_{\gamma}(x)^{p}=(\gamma \circ \psi)(x)$ for every $x \in \mathfrak{g}$. Then the $\mathcal{T}$-actions on $\mathfrak{g}$ and $\mathfrak{g}_{\psi}$ uniquely extend to actions on $U_{\chi_{\gamma}}(\mathfrak{g})$ and $U_{0}\left(\mathfrak{g}_{\psi}\right) /(\{a-\gamma(a) 1 ; a \in \mathfrak{a}\})$, respectively.

(3) The canonical map $\mathfrak{g} \hookrightarrow \mathfrak{g}_{\psi}$ of ordinary Lie algebras defines a $\mathcal{T}$-equivariant isomorphism

$$
U_{\chi_{\gamma}}(\mathfrak{g}) \stackrel{\sim}{\longrightarrow} U_{0}\left(\mathfrak{g}_{\psi}\right) /(\{a-\gamma(a) 1 ; a \in \mathfrak{a}\})
$$

of $k$-algebras.

Proof. (1) Given $t \in \mathcal{T}$, we only have to check that the action of $t \in \mathcal{T}$ on $\mathfrak{g}_{\psi}$ commutes with the $p$-mapping. For $x \in \mathfrak{g}$ and $a \in \mathfrak{a}$ we have

$$
\begin{aligned}
(t .(x, a))^{[p]} & =(t . x, a)^{[p]}=\left((t . x)^{[p]}, \psi(t . x)+a^{[p]}\right)=\left(t . x^{[p]}, \psi(x)+a^{[p]}\right) \\
& =t \cdot\left(x^{[p]}, \psi(x)+a^{[p]}\right)=t \cdot(x, a)^{[p]},
\end{aligned}
$$

as desired.

(2) As noted earlier, the action of $\mathcal{T}$ on $\mathfrak{g}_{\psi}$ uniquely extends to an operation of $\mathcal{T}$ on $U_{0}\left(\mathfrak{g}_{\psi}\right)$ by automorphisms. Note that $\mathcal{T}$ fixes the generating set $\{a-\gamma(a) 1 ; a \in$ $\mathfrak{a}\}$. Accordingly, the ideal generated by this set is $\mathcal{T}$-stable, and the action of $\mathcal{T}$ on $U_{0}\left(\mathfrak{g}_{\psi}\right)$ factors through $\Lambda_{\gamma}:=U_{0}\left(\mathfrak{g}_{\psi}\right) /(\{a-\gamma(a) 1 ; a \in \mathfrak{a}\})$.

To verify the corresponding fact for $U_{\chi_{\gamma}}(\mathfrak{g})$, we observe that $\chi_{\gamma}(t . x)=\chi_{\gamma}(x)$ for every $t \in \mathcal{T}$ and $x \in \mathfrak{g}$.

(3) By definition of the $\mathcal{T}$-action on $\mathfrak{g}_{\psi}$, the canonical map $\iota: \mathfrak{g} \longrightarrow \mathfrak{g}_{\psi} ; x \mapsto$ $(x, 0)$ is $\mathcal{T}$-equivariant. The same applies to the canonical homomorphism $\mathfrak{g}_{\psi} \longrightarrow$ $\Lambda_{\gamma}$. Thus, the composite $\omega$ also enjoys this property. Thanks to [17, (8.2)] the unique homomorphism

$$
\Omega: U_{\chi_{\gamma}}(\mathfrak{g}) \longrightarrow \Lambda_{\gamma}
$$

satisfying $\left.\Omega\right|_{\mathfrak{g}}=\omega$ is in fact an isomorphism. In view of $\omega$ being $\mathcal{T}$-equivariant, the algebra $U_{\chi_{\gamma}}(\mathfrak{g})$ being generated by $\mathfrak{g}$, and $\mathcal{T}$ acting on $U_{\chi_{\gamma}}(\mathfrak{g})$ and $\Lambda_{\gamma}$ via automorphisms, the homomorphism $\Omega$ is also $\mathcal{T}$-equivariant.

Let $\psi: \mathfrak{g} \longrightarrow \mathfrak{a}$ be a $p$-semilinear map between restricted Lie algebras, and suppose $\mathfrak{a}$ to be abelian. Given $\gamma \in X(\mathfrak{a})$, we denote by $\bmod \left(\mathfrak{g}_{\psi}, 0, \gamma\right)$ the full subcategory of $\bmod \left(\mathfrak{g}_{\psi}, 0\right)$ consisting of those $U_{0}\left(\mathfrak{g}_{\psi}\right)$-modules $M$ satisfying $a . m=$ $\gamma(a) m$ for all $a \in \mathfrak{a}$. Since $\mathfrak{a}$ is contained in the center of $\mathfrak{g}_{\psi}$, every simple $U_{0}\left(\mathfrak{g}_{\psi}\right)$ module belongs to $\bmod \left(\mathfrak{g}_{\psi}, 0, \gamma\right)$ for a suitable $\gamma \in X(\mathfrak{a})$. The third part of the foregoing result implies in particular that the canonical restriction functor induces an equivalence

$$
\bmod \left(\mathfrak{g}_{\psi}, 0, \gamma\right) \longrightarrow \bmod \left(\mathfrak{g}, \chi_{\gamma}\right) ;\left.\quad M \mapsto M\right|_{\mathfrak{g}}
$$

of categories. We are now going to investigate this equivalence more closely in the case where $\mathcal{T}$ is a multiplicative group.

Let $\mathcal{T}$ be an algebraic group acting on $(\mathfrak{g},[p])$ via automorphisms, and suppose that $\chi \in\left(\mathfrak{g}^{*}\right)^{\mathcal{T}}$ is a $\mathcal{T}$-invariant linear form. We denote by $\bmod (\mathfrak{g}, \chi, \mathcal{T})$ the category of $\left(U_{\chi}(\mathfrak{g}), \mathcal{T}\right)$-modules. If $M$ and $N$ belong to $\bmod (\mathfrak{g}, \chi, \mathcal{T})$, then $\operatorname{Hom}_{U_{\chi}(\mathfrak{g})}(M, N) \subset \operatorname{Hom}_{k}(M, N)$ is readily seen to be a $\mathcal{T}$-submodule. Moreover, for each $N \in \bmod (\mathfrak{g}, \chi, \mathcal{T})$ and every $t \in \mathcal{T}(k)$ the multiplication by $t$ induces an auto-equivalence of the functor

$$
\operatorname{Hom}_{U_{\chi}(\mathfrak{g})}(-, N): \bmod (\mathfrak{g}, \chi, \mathcal{T}) \longrightarrow \bmod k .
$$


The general theory of effacable functors guarantees the existence of a unique extension of this action to the right derived functors. For reduced algebraic groups the derived functors are $\mathcal{T}$-modules. We shall also be concerned with the case where $\mathcal{T}$ is infinitesimal. In this situation the categories $\bmod (\mathfrak{g}, \chi, \mathcal{T})$ and $\bmod U_{\chi}(\mathfrak{g}) \sharp H(\mathcal{T})$ are equivalent, and the $H(\mathcal{T})$-action on $\operatorname{Hom}_{U_{\chi}(\mathfrak{g})}(-, N)$ uniquely extends to the derived functors (see [6, (III.5.2)] for more detail).

Our next result identifies the right derived functors of the above functor with the restriction of $\operatorname{Ext}_{U_{\chi}(\mathfrak{g})}^{n}(-, N)$ to $\bmod (\mathfrak{g}, \chi, \mathcal{T})$.

Lemma 2.3. Let $\mathcal{T}$ be a multiplicative algebraic group which is either infinitesimal or a torus. Suppose that $\mathcal{T}$ operates on $(\mathfrak{g},[p])$ via automorphisms, and let $\psi$ : $\mathfrak{g} \longrightarrow \mathfrak{a}$ be a p-semilinear map into an abelian restricted Lie algebra $\mathfrak{a}$ such that $\psi(t . x)=\psi(x)$ for every $t \in \mathcal{T}, x \in \mathfrak{g}$. Given $\gamma \in X(\mathfrak{a})$, let $M, N$ be $\left(U_{0}\left(\mathfrak{g}_{\psi}\right), \mathcal{T}\right)$ modules belonging to $\bmod \left(\mathfrak{g}_{\psi}, \gamma\right)$. Then $\mathcal{T}$ naturally acts on $\operatorname{Ext}_{U_{0}\left(\mathfrak{g}_{\psi}\right)}^{1}(M, N)$ and $\operatorname{Ext}_{U_{\chi_{\gamma}}(\mathfrak{g})}^{1}\left(\left.M\right|_{\mathfrak{g}},\left.N\right|_{\mathfrak{g}}\right)$, and we have an isomorphism

$$
\operatorname{Ext}_{U_{0}\left(\mathfrak{g}_{\psi}\right)}^{1}(M, N) \cong \operatorname{Ext}_{U_{\chi \gamma}(\mathfrak{g})}^{1}\left(\left.M\right|_{\mathfrak{g}},\left.N\right|_{\mathfrak{g}}\right)
$$

of $\mathcal{T}$-modules.

Proof. Assuming $\mathcal{T}$ to be a torus, we note that its character group $X(\mathcal{T})$ is a free abelian group of finite rank. Recall that $\bmod \left(\mathfrak{g}_{\psi}, 0\right)^{\text {gr }}$ and $\bmod \left(\mathfrak{g}, \chi_{\gamma}\right)^{\text {gr }}$ are the categories of finite dimensional $X(\mathcal{T})$-graded $U_{0}\left(\mathfrak{g}_{\psi}\right)$-modules and $U_{\chi_{\gamma}}(\mathfrak{g})$-modules, respectively. Thanks to $[30, \S 1]$ these categories are equivalent to the categories of $\left(U_{0}\left(\mathfrak{g}_{\psi}\right), \mathcal{T}\right)$-modules and $\left(U_{\chi_{\gamma}}(\mathfrak{g}), \mathcal{T}\right)$-modules, respectively. By Lemma 2.1 both categories have enough projective objects.

We shall show that the two extension groups naturally have structures of $\mathcal{T}$ modules. It suffices to treat the case of $U_{0}\left(\mathfrak{g}_{\psi}\right)$-modules. To that end, we let $\bmod k^{\text {gr }}$ be the category of $X(\mathcal{T})$-graded vector spaces, and consider the forgetful functor $\mathcal{F}: \bmod \left(\mathfrak{g}_{\psi}, 0\right)^{\mathrm{gr}} \longrightarrow \bmod \left(\mathfrak{g}_{\psi}, 0\right)$ as well as the contravariant functor $(-, N)$ : $\bmod \left(\mathfrak{g}_{\psi}, 0\right)^{\mathrm{gr}} \longrightarrow \bmod k \quad ; \quad X \mapsto \operatorname{Hom}_{U_{0}\left(\mathfrak{g}_{\psi}\right)}(X, N)$. According to [30, (1.6)] the functor $\mathcal{F}$ is exact and sends projectives to projectives. Thus, the Grothendieck spectral sequence $R^{n} \operatorname{Hom}_{U_{0}\left(\mathfrak{g}_{\psi}\right)}(-, N) \circ L_{m} \mathcal{F} \Rightarrow R^{n+m}(-, N)$ (cf. [35. (11.40)]) degenerates to isomorphisms

$$
R^{n}(-, N) \cong \operatorname{Ext}_{U_{0}\left(\mathfrak{g}_{\psi}\right)}^{n}(-, N) \circ \mathcal{F}
$$

so that

is the right derived functor of

$$
\bmod \left(\mathfrak{g}_{\psi}, 0\right)^{\mathrm{gr}} \longrightarrow \bmod k \quad ; \quad M \mapsto \operatorname{Ext}_{U_{0}\left(\mathfrak{g}_{\psi}\right)}^{n}(M, N)
$$

$$
\bmod \left(\mathfrak{g}_{\psi}, 0\right)^{\mathrm{gr}} \longrightarrow \bmod k^{\mathrm{gr}} ; M \mapsto \operatorname{Hom}_{U_{0}\left(\mathfrak{g}_{\psi}\right)}(M, N)
$$

Accordingly, we have a canonical $\mathcal{T}$-action on the extension groups, which corresponds to a $X(\mathcal{T})$-grading.

Our assertion now follows from the $\mathcal{T}$-equivariance of the isomorphism given in Lemma 2.2.

Now assume $\mathcal{T}$ to be infinitesimal, and let $\mathcal{F}: \bmod \left(U_{\chi_{\gamma}}(\mathfrak{g}) \sharp H(\mathcal{T})\right) \longrightarrow \bmod \left(\mathfrak{g}, \chi_{\gamma}\right)$ be the forgetful functor. Since $U_{\chi_{\gamma}}(\mathfrak{g}) \sharp H(\mathcal{T})$ is a free $U_{\chi_{\gamma}}(\mathfrak{g})$-module, the functor $\mathcal{F}$ sends projectives to projectives. We may now apply the above arguments to the functor

$$
(-, N): \bmod \left(U_{\chi_{\gamma}}(\mathfrak{g}) \sharp H(\mathcal{T})\right) \longrightarrow \bmod H(\mathcal{T}) \quad ; \quad M \mapsto \operatorname{Hom}_{U_{\chi_{\gamma}}(\mathfrak{g})}(M, N)
$$


to obtain an $H(\mathcal{T})$-structure on $\operatorname{Ext}_{U_{\chi_{\gamma}}(\mathfrak{g})}^{1}(M, N)$. Thus, our result follows in this case from the equivalence of $\bmod \mathcal{T}$ and $\bmod H(\mathcal{T})$.

\section{Galois aCtions}

In the sequel, $\mathcal{G}$ denotes an infinitesimal group over an algebraically closed field $k$ of characteristic $p>0$. By definition, the function algebra $\mathcal{O}(\mathcal{G})$ of the group scheme $\mathcal{G}$ is a local, commutative Hopf algebra (of dimension a power of $p$ ). As mentioned earlier, the dual Hopf algebra $H(\mathcal{G}):=\mathcal{O}(\mathcal{G})^{*}$ is the algebra of measures of $\mathcal{G}$. Its category $\bmod H(\mathcal{G})$ of finite dimensional modules is equivalent to the corresponding module category for $\mathcal{G}$. Recall that the group structure of the character group $X(\mathcal{G})=\operatorname{Alg}_{k}(H(\mathcal{G}), k)$ is given by the convolution product

$$
(\lambda * \mu)(h):=\sum_{(h)} \lambda\left(h_{(1)}\right) \mu\left(h_{(2)}\right) \quad \forall \lambda, \mu \in X(\mathcal{G}), h \in H(\mathcal{G}) .
$$

The commutative $p$-group $X(\mathcal{G})$ acts on $H(\mathcal{G})$ via automorphisms of the associative algebra $H(\mathcal{G})$ :

$$
\lambda \cdot h:=\sum_{(h)} \lambda\left(h_{(1)}\right) h_{(2)} \quad \text { for } \lambda \in X(\mathcal{G}), h \in H(\mathcal{G}) .
$$

Direct computation shows that this operation is induced by the contragredient action of $\mathcal{O}(\mathcal{G})$ on $H(\mathcal{G})=\mathcal{O}(\mathcal{G})^{*}$.

If $\mathcal{N} \triangleleft \mathcal{G}$ is a normal subgroup, then $X(\mathcal{G} / \mathcal{N})$ is isomorphic to the subgroup of those $\lambda \in X(\mathcal{G})$ that vanish on the two-sided ideal $H(\mathcal{G}) H(\mathcal{N})^{\dagger}$ of $H(\mathcal{G})$, generated by the augmentation ideal $H(\mathcal{N})^{\dagger} \subset H(\mathcal{N})$ of the Hopf algebra $H(\mathcal{N})$.

By definition, the order $\operatorname{ord}(\mathcal{G})$ of $\mathcal{G}$ is the dimension of $H(\mathcal{G})$. Thus, $\operatorname{ord}(\mathcal{G})=$ $\operatorname{ord}(\mathcal{N}) \operatorname{ord}(\mathcal{G} / \mathcal{N})$ for every normal subgroup $\mathcal{N} \triangleleft \mathcal{G}$.

We begin by providing general results concerning the case where the factor group $\mathcal{G} / \mathcal{N}$ is multiplicative. Aside from trigonalizable group schemes, this set-up naturally arises for infinitesimal groups with tame principal block (cf. [18]).

Proposition 3.1. Let $\mathcal{N} \subset \mathcal{G}$ be a normal subgroup of the infinitesimal group $\mathcal{G}$ such that $\mathcal{G} / \mathcal{N}$ is multiplicative. Then $H(\mathcal{N})=H(\mathcal{G})^{X(\mathcal{G} / \mathcal{N})}$.

Proof. Directly from the definition we have $H(\mathcal{N}) \subset H(\mathcal{G})^{X(\mathcal{G} / \mathcal{N})}$. Since $\mathcal{G} / \mathcal{N}$ is multiplicative, we obtain

$$
\operatorname{dim}_{k} H(\mathcal{G})=\operatorname{ord}(\mathcal{G})=\operatorname{ord}(\mathcal{G} / \mathcal{N}) \operatorname{ord}(\mathcal{N})=|X(\mathcal{G} / \mathcal{N})| \operatorname{dim}_{k} H(\mathcal{N})
$$

As $\mathcal{O}(\mathcal{G} / \mathcal{N})=k[X(\mathcal{G} / \mathcal{N})]$ is a Hopf subalgebra of $\mathcal{O}(\mathcal{G})$, it follows from [33, (2.6)] that $\mathcal{O}(\mathcal{G})$ is a free $k[X(\mathcal{G} / \mathcal{N})]$-module. However, being a Hopf algebra, $\mathcal{O}(\mathcal{G})$ is self-injective (cf. [32, (2.1.3)]), so that $H(\mathcal{G})$ is a projective $\mathcal{O}(\mathcal{G})$-module. Hence $H(\mathcal{G})$ is also a projective module for the local algebra $k[X(\mathcal{G} / \mathcal{N})]$ relative to the module structure given by $(*)$. Thus, $H(\mathcal{G})$ is a free $k[X(\mathcal{G} / \mathcal{N})]$-module, implying

$$
\operatorname{dim}_{k} H(\mathcal{G})^{X(\mathcal{G} / \mathcal{N})}=\frac{1}{|X(\mathcal{G} / \mathcal{N})|} \operatorname{dim}_{k} H(\mathcal{G})=\operatorname{dim}_{k} H(\mathcal{N})
$$

This shows that the inclusion $H(\mathcal{N}) \subset H(\mathcal{G})^{X(\mathcal{G} / \mathcal{N})}$ is in fact an equality.

Let $\Lambda$ be a $k$-algebra, and $G$ a finite group which acts on $\Lambda$ via automorphisms. We denote by $\Lambda[G]$ the skew group algebra of $G$ over $\Lambda$ and refer the reader to 
[3] concerning its basic properties. Following [2] we say that $\Lambda: \Lambda^{G}$ is a Galois extension if

(a) $\Lambda$ is a projective $\Lambda[G]$-generator, and

(b) for every simple $\Lambda$-module $S$, the restriction $\left.S\right|_{\Lambda^{G}}$ is semisimple.

In view of $[2,(1.2)]$ condition (a) ensures that the algebras $\Lambda[G]$ and $\Lambda^{G}$ are Morita equivalent.

Given $\lambda$ in $X(\mathcal{G})$, we denote by $k_{\lambda}$ the one dimensional $H(\mathcal{G})$-module with underlying $k$-space $k$ and action

$$
h . \alpha=\lambda(h) \alpha \quad \forall h \in H(\mathcal{G}), \alpha \in k .
$$

To obtain a strong connection between the module categories of $H(\mathcal{G})$ and $H(\mathcal{N})$, we consider the following operation of $X(\mathcal{G})$ on the $\operatorname{set} \operatorname{Irr}(\mathcal{G})$ of isoclasses of simple $H(\mathcal{G})$-modules:

$$
\lambda \cdot[S]:=\left[S \otimes_{k} k_{(\lambda \circ \eta)}\right] .
$$

Here $\eta: H(\mathcal{G}) \longrightarrow H(\mathcal{G})$ denotes the antipode of the cocommutative Hopf algebra $H(\mathcal{G})$. Note that this action amounts to twisting the operation of $S$ by the inverse of the algebra automorphism of $H(\mathcal{G})$ effected by $\lambda$.

Remark. Adopting the hypotheses of Proposition 3.1, let $\mathcal{B} \subset H(\mathcal{G})$ be a block of $H(\mathcal{G})$. If there exists a simple $\mathcal{B}$-module $S$ such that $\lambda \cdot[S]$ belongs to $\mathcal{B}$ for all characters $\lambda \in X(\mathcal{G} / \mathcal{N})$, then the central idempotent defining $\mathcal{B}$ is a fixed-point and $H(\mathcal{G})=\mathcal{B} \oplus \mathcal{C}$ decomposes into a direct sum of $X(\mathcal{G} / \mathcal{N})$-invariant block ideals. (The term block ideal is used to refer to a direct sum of blocks.) Accordingly, we also have a decomposition

$$
H(\mathcal{N})=\mathcal{B}^{X(\mathcal{G} / \mathcal{N})} \oplus \mathcal{C}^{X(\mathcal{G} / \mathcal{N})}
$$

of $H(\mathcal{N})$ into a sum of block ideals.

Given an $H(\mathcal{G})$-module $M$ and $\lambda \in X(\mathcal{G})$, we put

$$
M_{\lambda}:=\{m \in M ; h . m=\lambda(h) m \forall h \in H(\mathcal{G}), m \in M\} .
$$

Let $\mathcal{N} \subset \mathcal{G}$ be a subgroup. The restriction of $M$ to the Hopf algebra $H(\mathcal{N}) \subset H(\mathcal{G})$ will be denoted $\left.M\right|_{\mathcal{N}}$.

In view of [2, (5.1)] our next result provides a simple criterion for $H(\mathcal{G}): H(\mathcal{N})$ to be a Galois extension.

Proposition 3.2. Let $\mathcal{G}$ be an infinitesimal group, and $\mathcal{N} \triangleleft \mathcal{G}$ a normal subgroup such that $\mathcal{G} / \mathcal{N}$ is multiplicative. Then the following statements are equivalent:

(1) The group $X(\mathcal{G} / \mathcal{N})$ acts freely on $\operatorname{Irr}(\mathcal{G})$.

(2) For every simple $H(\mathcal{G})$-module $S$, the module $\left.S\right|_{\mathcal{N}}$ is simple.

Proof. (1) $\Rightarrow(2)$. This follows from Proposition 3.1 and the proof of [2, (5.1)].

$(2) \Rightarrow(1)$. Let $S$ be a simple $H(\mathcal{G})$-module. We let $H(\mathcal{G})$ act on $\operatorname{Hom}_{k}(S, S)$ via

$$
(h \cdot \varphi)(s):=\sum_{(h)} h_{(1)} \varphi\left(\eta\left(h_{(2)}\right) s\right) \quad \forall h \in H(\mathcal{G}), \varphi \in \operatorname{Hom}_{k}(S, S), s \in S .
$$

Given $\lambda \in X(\mathcal{G} / \mathcal{N})$, direct computation shows

$$
\operatorname{Hom}_{H(\mathcal{G})}\left(S, S \otimes_{k} k_{\lambda \circ \eta}\right)=\operatorname{Hom}_{k}(S, S)_{\lambda} .
$$

Since $\mathcal{N} \triangleleft \mathcal{G}$ is a normal subgroup, $\operatorname{Hom}_{H(\mathcal{N})}(S, S)$ is an $H(\mathcal{G})$-submodule of $\operatorname{Hom}_{k}(S, S)$. In view of $H(\mathcal{N})^{\dagger} \subset \operatorname{ker} \lambda$, we actually have

$$
\operatorname{Hom}_{H(\mathcal{G})}\left(S, S \otimes_{k} k_{\lambda \circ \eta}\right)=\operatorname{Hom}_{H(\mathcal{N})}(S, S)_{\lambda} .
$$


Thus, the assumption $[S]=\lambda \cdot[S]$ implies $\operatorname{Hom}_{H(\mathcal{N})}(S, S)_{\lambda} \neq(0)$. On the other hand, the module $\left.S\right|_{\mathcal{N}}$ is simple, and Schur's Lemma yields

$$
\operatorname{Hom}_{H(\mathcal{N})}(S, S)=k \cdot \operatorname{id}_{S}=\operatorname{Hom}_{H(\mathcal{N})}(S, S)_{\varepsilon},
$$

so that $\lambda=\varepsilon$, as desired.

For future reference we record the following subsidiary result concerning free Galois actions.

Lemma 3.3. Let $\Lambda$ be a k-algebra, and $G$ a finite group which acts on $\Lambda$ via automorphisms such that the induced operation on $\operatorname{Irr}(\Lambda)$ is free. Then the canonical restriction functor $\operatorname{Res}_{\Lambda^{G}}^{\Lambda}: \bmod \Lambda \longrightarrow \bmod \Lambda^{G}$ is length-preserving and sends principal indecomposables to principal indecomposables.

Proof. According to [2, (1.2)] the functor

$$
\text { Fix : } \bmod \Lambda[G] \longrightarrow \bmod \Lambda^{G} ; M \mapsto M^{G}
$$

is an equivalence of categories. As such, it has the properties stated in our lemma. Direct computation shows that

$$
\operatorname{Res}_{\Lambda^{G}}^{\Lambda}=\operatorname{Fix} \circ \operatorname{Ind}_{\Lambda}^{\Lambda[G]}
$$

is the composite of Fix with the canonical induction functor

$$
\operatorname{Ind}_{\Lambda}^{\Lambda[G]}: \bmod \Lambda \longrightarrow \bmod \Lambda[G] \quad ; \quad M \mapsto \Lambda[G] \otimes_{\Lambda} M .
$$

Since $\Lambda[G]$ is a free right $\Lambda$-module, $\operatorname{Ind}_{\Lambda}^{\Lambda[G]}$ is exact and sends projectives to projectives. As $G$ acts freely on $\operatorname{Irr}(\Lambda)$, the arguments of [2, (5.1)] show that the module $\operatorname{Ind}_{\Lambda}^{\Lambda[G]}(S)$ is simple, whenever $S \in \bmod \Lambda$ is. Accordingly, $\operatorname{Ind}_{\Lambda}^{\Lambda[G]}$ is length-preserving. Finally, let $P$ be a principal indecomposable $\Lambda$-module, and let $X, Y$ be $\Lambda[G]$-modules such that $\operatorname{Ind}_{\Lambda}^{\Lambda[G]}(P)=X \oplus Y$. Then we have

$$
\left.\left.\left.X\right|_{\Lambda} \oplus Y\right|_{\Lambda} \cong \operatorname{Ind}_{\Lambda}^{\Lambda[G]}(P)\right|_{\Lambda} \cong \bigoplus_{g \in G} g \cdot P,
$$

where $g . P$ is the $\Lambda$-module with underlying $k$-space $P$ and action

$$
\lambda . x=\left(g^{-1} \cdot \lambda\right) x \quad \forall g \in G, x \in P .
$$

As $G$ acts freely on the simples, the constituents of the right-hand sum are pairwise non-isomorphic. Thus, if $X \neq(0)$, then the theorem of Krull-Remak-Schmidt provides a subset $H \subset G$ such that $\left.X\right|_{\Lambda} \cong \bigoplus_{h \in H}$ h.P. As $X$ is a $\Lambda[G]$-module, we have $X \cong g$. $X$ for every $g \in G$. Accordingly, $H=G$ and $X=\operatorname{Ind}_{\Lambda}^{\Lambda[G]}(P)$. Consequently, $\operatorname{Ind}_{\Lambda}^{\Lambda[G]}(P)$ is a principal indecomposable module.

If $\mathcal{G}$ is an algebraic $k$-group we denote by $\mathcal{G}_{r}$ the kernel of the $r$-th iterate of the Frobenius homomorphism of $\mathcal{G}$. An infinitesimal $k$-group $\mathcal{G}$ satisfies $\mathcal{G}=\mathcal{G}_{r}$ for some $r \geq 0$, and the minimal such number ht $(\mathcal{G})$ is called the height of $\mathcal{G}$.

In the sequel, we denote by $\mathcal{M}(\mathcal{G})$ the multiplicative center of $\mathcal{G}$, that is, the unique largest multiplicative normal subgroup of the infinitesimal group $\mathcal{G}$. The group $\mathcal{M}(\mathcal{G})$ is contained in the center $\operatorname{Cent}(\mathcal{G})$ of $\mathcal{G}$ (cf. [9, (II,§1,3.9), (IV, $\S 1,4.4)$ ] for more details).

In the following we let $\mathcal{B}_{0}(\mathcal{G})$ be the principal block of $H(\mathcal{G})$. By definition, $\mathcal{B}_{0}(\mathcal{G})$ is the unique block which is not annihilated by the co-unit $\varepsilon$ of $H(\mathcal{G})$. 
Suppose that $p \geq 3$ and let $T \subset \mathrm{SL}(2)$ be the standard maximal torus of diagonal matrices. Given $r \geq 1$, we put $\mathcal{Q}_{[r]}:=\mathrm{SL}(2)_{1} T_{r}$. According to [20, (5.5)] the groups $\mathcal{Q}_{[r]}$ are precisely those semisimple infinitesimal groups whose distribution algebras possess a tame principal block. The Lie algebra $\operatorname{Lie}\left(\mathcal{Q}_{[r]}\right)$ is the special linear Lie algebra $\mathfrak{s l}(2)$ of trace zero $(2 \times 2)$-matrices. We let $\{e, h, f\}$ be the standard basis of $\mathfrak{s l}(2)$, that is,

$$
e:=\left(\begin{array}{ll}
0 & 1 \\
0 & 0
\end{array}\right) \quad ; \quad h:=\left(\begin{array}{cc}
1 & 0 \\
0 & -1
\end{array}\right) \quad ; \quad f:=\left(\begin{array}{ll}
0 & 0 \\
1 & 0
\end{array}\right) .
$$

Given a $k$-vector space $V$, we denote by $V^{(1)}$ the $k$-space with multiplication given by $\alpha . v=\alpha^{\frac{1}{p}} v$. A $p$-semilinear map $\psi: \mathfrak{s l}(2) \longrightarrow \mathfrak{a}$ may thus also be interpreted as a linear map $\psi: \mathfrak{s l}(2)^{(1)} \longrightarrow \mathfrak{a}$. We call $\psi$ semisimple if $k e \oplus k f \subset \operatorname{ker} \psi$. Thus, letting $T \times \mathfrak{s l}(2) \longrightarrow \mathfrak{s l}(2) ;(t, x) \mapsto t . x$ denote the adjoint action of the standard maximal torus $T \subset \mathrm{SL}(2)$ on $\mathfrak{s l}(2)$, the semisimple $p$-semilinear maps are those satisfying $\psi(t . x)=\psi(x)$ for $t \in T$ and $x \in \mathfrak{s l}(2)$.

Recall from Section 1 the definition of the restricted Lie algebra $\mathfrak{s l}(2)_{\psi}:=\mathfrak{s l}(2) \oplus$ $\mathfrak{a}$, with multiplication and $p$-map given by

$[(x, a),(y, b)]=([x, y], 0)$ and $(x, a)^{[p]}=\left(x^{[p]}, \psi(x)+a^{[p]}\right) \quad \forall x, y \in \mathfrak{s l}(2), a, b \in \mathfrak{a}$, respectively.

As usual, we let $C(\mathfrak{g}):=\{x \in \mathfrak{g} ;[x, y]=0\}$ be the center of the restricted Lie algebra $(\mathfrak{g},[p])$.

Lemma 3.4. Let $\mathcal{G}$ be an infinitesimal group of characteristic $p \geq 3$ with Lie algebra $\mathfrak{g}:=\operatorname{Lie}(\mathcal{G})$. If $\mathcal{B}_{0}(\mathcal{G})$ is tame and $\operatorname{ht}(\mathcal{G} / \mathcal{M}(\mathcal{G})) \geq 2$, then there exists a semisimple p-semilinear map $\psi: \mathfrak{s l}(2) \longrightarrow C(\mathfrak{g})$ such that $\mathfrak{g} \cong \mathfrak{s l}(2)_{\psi}$ is a central extension of $\mathfrak{s l}(2)$.

Proof. A consecutive application of Theorem 1.2 and [20, (6.4)] provides a $p$ semilinear map $\psi: \mathfrak{s l}(2) \longrightarrow C(\mathfrak{g})$ such that $\mathfrak{g} \cong \mathfrak{s l}(2)_{\psi}$. According to [18, (3.3)] the map $\psi$ gives rise to a homomorphism

$$
\psi: \mathfrak{s l}(2)^{(1)} \longrightarrow C(\mathfrak{g})
$$

of $\mathcal{G}$-modules (relative to the adjoint representation). Since the center of $\mathcal{G}$ acts trivially on both modules, $\psi$ is in fact a homomorphism for the group $\mathcal{G} / \operatorname{Cent}(\mathcal{G})$, which, by Theorem 1.1, is isomorphic to some $\mathcal{Q}_{[r]}$. In particular, the abelian normal subgroup $\operatorname{Cent}\left(\mathcal{G}_{1}\right)$ of $\mathcal{G}$ is contained in $\operatorname{Cent}(\mathcal{G})$, so that $\mathcal{G}$ operates trivially on $C(\mathfrak{g})$.

Let $\mathfrak{s l}(2)=\mathfrak{s l}(2)_{\alpha} \oplus \mathfrak{s l}(2)_{0} \oplus \mathfrak{s l}(2)_{-\alpha}=k e \oplus k h \oplus k f$ be the root space decomposition of $\mathfrak{s l}(2)$ relative to the the multiplicative subgroup $T_{r}$ of $\mathcal{Q}_{[r]}$. Since $\mathcal{Q}_{[r]}$ and $\mathfrak{s l}(2)$ are defined over the Galois field $\mathbb{F}_{p}$, an application of [29, (I.9.10)] shows that $\mathfrak{s l}(2)^{(1)}$ is isomorphic to the Frobenius twist of $\mathfrak{s l}(2)$. Accordingly, $\left(\mathcal{Q}_{[r]}\right)_{1}=\mathrm{SL}(2)_{1}$ operates trivially on $\mathfrak{s l}(2)^{(1)}$, and the weights are just $-p \alpha, 0, p \alpha$. Since $p \neq 2$, the character $\alpha$ has order $p^{r}$. Thus, $\beta:=p \alpha$ has order $p^{r-1}$, and the weight space decomposition of $\mathfrak{s l}(2)^{(1)}$ is given by

$$
\mathfrak{s l}(2)^{(1)}=\mathfrak{s l}(2)_{\beta}^{(1)} \oplus \mathfrak{s l}(2)_{0}^{(1)} \oplus \mathfrak{s l}(2)_{-\beta}^{(1)} .
$$

Thanks to Theorem 1.2 and [18, $(1.5(2))]$ our assumption on the height of $\mathcal{G} / \mathcal{M}(\mathcal{G})$ implies $r \geq 2$, so that $\beta \neq 0$. As $T_{r}$ acts trivially on $C(\mathfrak{g})$, the root vectors $\{e, f\}$ are annihilated by $\psi$, and $\psi$ is semisimple. 
Proposition 3.5. Let $\mathcal{G}$ be an infinitesimal group of characteristic $p \geq 3$ such that $\mathcal{B}_{0}(\mathcal{G})$ is tame. Then the following statements hold:

(1) There exists a quotient map $\pi: \mathcal{G} \longrightarrow \mathcal{Q}_{[r]}$ such that $\operatorname{ker} \pi=\operatorname{Cent}(\mathcal{G})$.

(2) There exists a multiplicative subgroup $\mathcal{M} \subset \mathcal{G}$ such that

(a) $\mathcal{G}=\mathcal{G}_{1} \mathcal{M}$, and

(b) $\left.\pi\right|_{\mathcal{M}}: \mathcal{M} \longrightarrow T_{r}$ is a quotient map with kernel $\mathcal{M}(\mathcal{G})$.

Proof. (1) This is a direct consequence of Theorem 1.1.

(2) We set $\mathcal{G}^{\prime}:=\mathcal{G} / \mathcal{M}(\mathcal{G})$, and let $\pi^{\prime}: \mathcal{G}^{\prime} \longrightarrow \mathcal{Q}_{[r]}$ be the quotient map induced by $\pi$. Thanks to [18, (6.3)] there exists a closed embedding $\sigma: T_{r} \longrightarrow \mathcal{G}^{\prime}$ such that $\pi^{\prime} \circ \sigma=\operatorname{id}_{T_{r}}$ and $\mathcal{G}^{\prime}=\mathcal{G}_{1}^{\prime} \sigma\left(T_{r}\right)$. Letting $\eta: \mathcal{G} \longrightarrow \mathcal{G}^{\prime}$ be the canonical quotient map, we put $\mathcal{M}:=\eta^{-1}\left(\sigma\left(T_{r}\right)\right)$. Owing to $[9$, $(\mathrm{IV}, \S 1,4.5)]$ the group $\mathcal{M}$ is, as an extension of two connected multiplicative groups, multiplicative.

It was shown in the proof of Theorem 1.2 that $\eta$ induces a quotient map $\mathcal{G}_{1} \longrightarrow$ $\mathcal{G}_{1}^{\prime}$. Accordingly, the foregoing observations ensure that

$$
\left.\eta\right|_{\mathcal{G}_{1} \mathcal{M}}: \mathcal{G}_{1} \mathcal{M} \longrightarrow \mathcal{G}^{\prime}
$$

is a quotient map with kernel $\mathcal{M}(\mathcal{G})$. Consequently, the groups $\mathcal{G}_{1} \mathcal{M}$ and $\mathcal{G}$ have the same order, so that $\mathcal{G}=\mathcal{G}_{1} \mathcal{M}$.

By definition of $\mathcal{M}$, the homomorphism $\eta$ induces a quotient map $\left.\eta\right|_{\mathcal{M}}: \mathcal{M} \longrightarrow$ $\sigma\left(T_{r}\right)$ with kernel $\mathcal{M}(\mathcal{G})$. Since $\pi^{\prime} \circ \sigma=\mathrm{id}_{T_{r}}$, the restriction $\left.\pi^{\prime}\right|_{\sigma\left(T_{r}\right)}: \sigma\left(T_{r}\right) \longrightarrow T_{r}$ is an isomorphism. Thus, $\left.\pi\right|_{\mathcal{M}}=\left.\left.\pi^{\prime}\right|_{\sigma\left(T_{r}\right)} \circ \eta\right|_{\mathcal{M}}$ inherits the desired properties from $\left.\eta\right|_{\mathcal{M}}$

For $r \geq 1$, we consider the multiplicative group $\mu_{p^{r}}:=\operatorname{Spec}_{k}\left(k[T] /\left(T^{p^{r}}-1\right)\right)$, which associates to every commutative $k$-algebra $R$ the group

$$
\mu_{p^{r}}(R):=\left\{x \in R ; x^{p^{r}}=1\right\} .
$$

By general theory, every infinitesimal multiplicative group is a product of groups of type $\mu_{p^{r}}$. Directly from the definition we obtain the isomorphism $X\left(\mu_{p^{r}}\right) \cong \mathbb{Z} /\left(p^{r}\right)$.

Lemma 3.6. Let $\mathcal{G}$ be an infinitesimal group of characteristic $p \geq 3$ such that $\mathcal{B}_{0}(\mathcal{G})$ is tame. Then $\mathcal{G} / \mathcal{G}_{1} \mathcal{M}(\mathcal{G}) \cong \mu_{p^{r-1}}$, where $r=\operatorname{ht}(\mathcal{G} / \mathcal{M}(\mathcal{G}))$.

Proof. We put $\mathcal{G}^{\prime}:=\mathcal{G} / \mathcal{M}(\mathcal{G})$ as well as $\mathcal{N}:=\mathcal{G}_{1} \mathcal{M}(\mathcal{G})$. According to [18, (2.1)] we have $\mathcal{G}^{\prime} / \mathcal{G}_{1}^{\prime} \cong \mu_{p^{r-1}}$. The canonical quotient map $\mathcal{G} \longrightarrow \mathcal{G}^{\prime} / \mathcal{G}_{1}^{\prime}$ thus induces a quotient map

$$
\pi: \mathcal{G} / \mathcal{N} \longrightarrow \mu_{p^{r-1}}
$$

Owing to Theorem 1.2 the map $\mathcal{G} \longrightarrow \mathcal{G}^{\prime}$ induces a quotient map $\mathcal{G}_{1} \longrightarrow \mathcal{G}_{1}^{\prime}$ with kernel $\mathcal{M}(\mathcal{G}) \cap \mathcal{G}_{1}=\mathcal{M}\left(\mathcal{G}_{1}\right)$. Consequently,

$$
\begin{aligned}
\operatorname{ord}(\mathcal{G} / \mathcal{N}) & =\frac{\operatorname{ord}(\mathcal{G})}{\operatorname{ord}(\mathcal{N})}=\frac{\operatorname{ord}(\mathcal{G}) \operatorname{ord}\left(\mathcal{M}\left(\mathcal{G}_{1}\right)\right)}{\operatorname{ord}(\mathcal{M}(\mathcal{G})) \operatorname{ord}\left(\mathcal{G}_{1}\right)}=\frac{\operatorname{ord}(\mathcal{G})}{\operatorname{ord}\left(\mathcal{M}(\mathcal{G}) \operatorname{ord}\left(\mathcal{G}_{1}^{\prime}\right)\right.} \\
& =\frac{\operatorname{ord}\left(\mathcal{G}^{\prime}\right)}{\operatorname{ord}\left(\mathcal{G}_{1}^{\prime}\right)}=p^{r-1}
\end{aligned}
$$

so that the quotient map $\pi$ is in fact an isomorphism.

As will be shown later, the block structure of the distribution algebra of an infinitesimal group $\mathcal{G}$ with tame principal block is largely determined by the normal subgroup

$$
\mathcal{N}(\mathcal{G}):=\mathcal{G}_{1} \mathcal{M}(\mathcal{G})
$$


Our next result is crucial as it establishes a particularly useful Galois correspondence between $\mathcal{N}(\mathcal{G})$ and $\mathcal{G}$, which will serve in our context as a substitute for the well-known Brauer correspondence between the principal blocks of a finite group $G$ and its local subgroup $P C_{G}(P)$, defined by a Sylow-p-subgroup $P \subset G$.

In the sequel we will identify the algebra $H(\mathcal{G})$ of distributions of an infinitesimal group $\mathcal{G}$ of height $\leq 1$ with the restricted enveloping algebra $U_{0}(\mathfrak{g})$ of its Lie algebra $\mathfrak{g}=\operatorname{Lie}(\mathcal{G})$ (cf. [9, $(\mathrm{II}, \S 7,4.2)])$.

Theorem 3.7. Let $\mathcal{G}$ be an infinitesimal group of characteristic $p \geq 3$ with tame principal block. Then the following statements hold:

(1) The restriction $\left.S\right|_{\mathcal{G}_{1}}$ of every simple $\mathcal{G}$-module $S$ is simple.

(2) The character group $X(\mathcal{G} / \mathcal{N}(\mathcal{G})$ ) operates freely on the set of isoclasses of simple $H(\mathcal{G})$-modules, and $H(\mathcal{G}): H(\mathcal{N}(\mathcal{G}))$ is a Galois extension with Galois group $X(\mathcal{G} / \mathcal{N}(\mathcal{G}))$.

(3) We have an isomorphism $X(\mathcal{G} / \mathcal{N}(\mathcal{G})) \cong \mathbb{Z} /\left(p^{r-1}\right)$, where $r:=\operatorname{ht}(\mathcal{G} / \mathcal{M}(\mathcal{G}))$.

Proof. By Proposition 3.5 there exists a quotient map $\pi: \mathcal{G} \longrightarrow \mathcal{Q}_{[s]}$ with kernel $\operatorname{Cent}(\mathcal{G})$. Thanks to [18, (2.1)] we obtain $s=r$. If $r=1$, then Lemma 3.6 yields $\mathcal{G}_{1} \mathcal{M}(\mathcal{G})=\mathcal{G}$, and there is nothing to be shown. Alternatively, Proposition 3.5 provides a multiplicative subgroup $\mathcal{M} \subset \mathcal{G}$ such that

(a) $\mathcal{G}=\mathcal{G}_{1} \mathcal{M}$, and

(b) $\left.\pi\right|_{\mathcal{M}}: \mathcal{M} \longrightarrow T_{r}$ is a quotient map with kernel $\mathcal{M}(\mathcal{G})$.

As before, $T \subset \mathrm{SL}(2)$ denotes the standard maximal torus of diagonal matrices. We put $\mathcal{N}:=\mathcal{N}(\mathcal{G})$ as well as $\hat{\mathcal{G}}:=\mathcal{G}_{1} \rtimes \mathcal{M}$ and $\hat{\mathcal{N}}:=\mathcal{G}_{1} \times \mathcal{M}(\mathcal{G})$. There results a commutative diagram

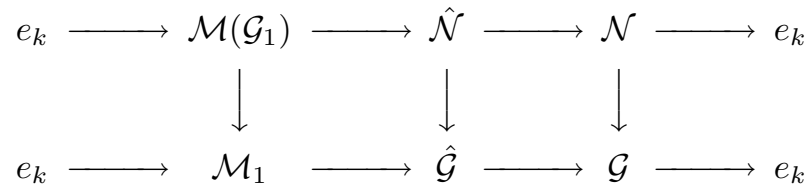

with exact rows and injective vertical arrows.

(i) Every simple $\mathcal{G}_{1}$-module has the structure of a $\hat{\mathcal{G}}$-module.

Let $\mathfrak{g}:=\operatorname{Lie}(\mathcal{G})$ be the Lie algebra of $\mathcal{G}$. According to Lemma 3.4 there exists a semisimple $p$-semilinear map $\psi: \mathfrak{s l}(2) \longrightarrow C(\mathfrak{g})$ such that $\mathfrak{g}=\mathfrak{s l}(2)_{\psi}$. We let $T$ act on $\mathfrak{g}=\mathfrak{s l}(2)_{\psi}$ via

$$
t .(x, c):=(\operatorname{Ad}(t)(x), c) \quad \forall t \in T, x \in \mathfrak{s l}(2), c \in C(\mathfrak{g}) .
$$

If $S$ is a simple $\mathcal{G}_{1}$-module, then $S$ is a simple $U_{0}(\mathfrak{g}) \cong H\left(\mathcal{G}_{1}\right)$-module (cf. 9, $(\mathrm{II}, \S 7,4.2)])$, and Lemma 2.1 provides a $\left(U_{0}(\mathfrak{g}), T\right)$-structure for $S$. We consider the action of $T$ on $\mathcal{G}_{1}$ that corresponds to the action of $T$ on $\mathfrak{g}$, and note that $S$ has the structure of a $\left(\mathcal{G}_{1} \rtimes T\right)$-module.

The group $\mathcal{M}$ operates on $\mathfrak{g}$ via the adjoint representation. Since $C(\mathfrak{g})=$ $\operatorname{Lie}(\operatorname{Cent}(\mathcal{G}))(\operatorname{cf}$. Theorem 1.1) and $\mathfrak{s l}(2)=[\mathfrak{g}, \mathfrak{g}]$, the decomposition

$$
\mathfrak{g}=\mathfrak{s l}(2) \oplus C(\mathfrak{g})
$$

is a decomposition of $\mathcal{M}$-modules, with $\mathcal{M}$ acting on $\mathfrak{g}$ via

$$
m(x, c)=(\operatorname{Ad}(m)(x), c) \quad \forall m \in \mathcal{M}, x \in \mathfrak{s l}(2), c \in C(\mathfrak{g}) .
$$

The action of $\mathcal{M}$ is thus determined by that of its image $T_{r}$ in $\mathcal{Q}_{[r]}$. Accordingly, the operation of $T_{r} \cong \mathcal{M} / \mathcal{M}(\mathcal{G})$ on $\mathfrak{g}$ coincides with the restriction of the $T$-action 
on $\mathfrak{g}$. It follows that the pull-back along the quotient map

$$
\hat{\mathcal{G}} \longrightarrow \mathcal{G}_{1} \rtimes T_{r} \quad ; \quad(g, m) \mapsto(g, \pi(m)),
$$

provides $S$ with the structure of a $\hat{\mathcal{G}}$-module such that $\left.S\right|_{\mathcal{G}_{1}}$ is the originally given module.

(ii) Let $S$ be a simple $\hat{\mathcal{G}}$-module. Then $\left.S\right|_{\mathcal{G}_{1}}$ is simple.

Let $S$ be a simple $\hat{\mathcal{G}}$-module, and let $\left.S^{\prime} \subset S\right|_{\mathcal{G}_{1}}$ be a simple $\mathcal{G}_{1}$-submodule. Thanks to (i), the space $S^{\prime}$ also has a $\hat{\mathcal{G}}$-structure. By assumption, the $\left(\hat{\mathcal{G}} / \mathcal{G}_{1}\right)$ module $\operatorname{Hom}_{\mathcal{G}_{1}}\left(S^{\prime}, S\right)$ is not zero. Since the factor group $\hat{\mathcal{G}} / \mathcal{G}_{1}$ is multiplicative, there exists a character $\omega \in X\left(\hat{\mathcal{G}} / \mathcal{G}_{1}\right)$ such that $\operatorname{Hom}_{\mathcal{G}_{1}}\left(S^{\prime}, S\right)_{\omega} \neq(0)$. By the proof of Proposition 3.2 we can find a non-zero homomorphism $S^{\prime} \longrightarrow S \otimes_{k} k_{\omega \circ \eta}$ of $\hat{\mathcal{G}}$-modules. Consequently, these two simple $\hat{\mathcal{G}}$-modules are isomorphic, and the restriction $\left.\left.S\right|_{\mathcal{G}_{1}} \cong S^{\prime}\right|_{\mathcal{G}_{1}}$ is simple.

Since every simple $\mathcal{G}$-module has, via pull-back, the structure of a simple $\hat{\mathcal{G}}$ module, assertion (1) is a direct consequence of (ii).

(iii) The group $X(\mathcal{G} / \mathcal{N})$ operates freely on the isoclasses of simple $\mathcal{G}$-modules.

Since $\mathcal{G}_{1} \subset \mathcal{N}$, part (1) implies that the restriction $\left.S\right|_{\mathcal{N}}$ of every simple $\mathcal{G}$ module $S$ is simple. We may now apply Lemma 3.6 and Proposition 3.2 to obtain our assertion.

Thanks to Lemma 3.6 the factor group $\mathcal{G} / \mathcal{N}$ is multiplicative. A consecutive application of [2, (5.1)] and Proposition 3.1 now shows that $H(\mathcal{G}): H(\mathcal{N})$ is a Galois extension. Another application of Lemma 3.6 yields $X(\mathcal{G} / \mathcal{N}) \cong X\left(\mu_{p^{r-1}}\right) \cong$ $\mathbb{Z} /\left(p^{r-1}\right)$.

Corollary 3.8. Let $\mathcal{G}$ be an infinitesimal group of characteristic $p \geq 3$. If the principal block of $H(\mathcal{G})$ is tame, then $H(\mathcal{G}): H\left(\mathcal{G}_{1}\right)$ is a Galois extension, whose Galois group $X\left(\mathcal{G} / \mathcal{G}_{1}\right)$ is a product of cyclic p-groups that acts freely on $\operatorname{Irr}(\mathcal{G})$.

Proof. Since the group $\mathcal{G} / \mathcal{G}_{1}$ is an extension of the connected multiplicative groups $\mathcal{G} / \mathcal{N}(\mathcal{G})$ and $\mathcal{N}(\mathcal{G}) / \mathcal{G}_{1}$ (cf. [9, (III, $\left.\left.\S 3,3.7\right)\right]$ ), it is multiplicative (cf. [9, $\left.(\mathrm{IV}, \S 1,4.5)\right]$ ). The desired result now follows from a consecutive application of Theorem $\underline{3.7}(1)$, Propositions 3.2, 3.1 and [2, (5.1)].

\section{Block Representation type}

Throughout this section, we let $k$ be an algebraically closed field of characteristic $p \geq 3$. In 18 those groups $\mathcal{G}$ with trivial multiplicative center, having a tame principal block $\mathcal{B}_{0}(\mathcal{G}) \subset H(\mathcal{G})$, were determined. Moreover, in that case the block structure of $H(\mathcal{G})$ is also completely understood. By results of [17, the presence of a multiplicative center may complicate the representation theory of $H(\mathcal{G})$ significantly. However, as the main result of this section, Theorem 4.3, shows, this phenomenon is essentially a feature of infinitesimal groups of height 1 .

Our first subsidiary result implies that the representation theory of the normal subgroup $\mathcal{N}(\mathcal{G}) \triangleleft \mathcal{G}$ essentially coincides with that of the first Frobenius kernel $\mathcal{G}_{1}$.

Lemma 4.1. Let $\mathcal{G}$ be an infinitesimal $k$-group, and $\mathcal{K} \triangleleft \mathcal{G}$ a normal subgroup such that $\mathcal{G}=\mathcal{K} \mathcal{M}(\mathcal{G})$. Then the following statements hold:

(1) There exists a natural number $n \in \mathbb{N}$ such that $H(\mathcal{G})$ is a block ideal of $H(\mathcal{K})^{n}$, with each block of $H(\mathcal{K})$ occurring as a block of $H(\mathcal{G})$.

(2) If $\mathrm{nb}_{\mathcal{G}}$ and $\mathrm{nb}_{\mathcal{K}}$ denote the number of blocks of $H(\mathcal{G})$ and $H(\mathcal{K})$, respectively, then we have $\operatorname{nb}_{\mathcal{G}}=\operatorname{nb}_{\mathcal{K}} \operatorname{ord}(\mathcal{M}(\mathcal{G}) / \mathcal{M}(\mathcal{K}))$. 
Proof. (1) Let $\mathcal{L} \subset \mathcal{K} \times \mathcal{M}(\mathcal{G})$ be the kernel of the quotient map

$$
\mathcal{K} \times \mathcal{M}(\mathcal{G}) \longrightarrow \mathcal{G}
$$

that is induced by the multiplication of $\mathcal{G}$. Since $\mathcal{K}$ is normal in $\mathcal{G}$ and $\mathcal{M}(\mathcal{K})$ is a characteristic subgroup of $\mathcal{K}$, it follows that $\mathcal{M}(\mathcal{K})$ is normal in $\mathcal{G}$ (cf. 9, $(\mathrm{II}, \S 1,3.9),(\mathrm{IV}, \S 3,1.1)])$. Thus, $\mathcal{M}(\mathcal{K}) \subset \mathcal{M}(\mathcal{G})$, and $\mathcal{M}(\mathcal{K})=\mathcal{K} \cap \mathcal{M}(\mathcal{G})$. As a result,

$$
\mathcal{L}=\left\{\left(g, g^{-1}\right) ; g \in \mathcal{M}(\mathcal{K})\right\} \cong \mathcal{M}(\mathcal{K})
$$

is a multiplicative, normal subgroup of $\mathcal{K} \times \mathcal{M}(\mathcal{G})$. According to the proof of 20, $(1.1(1))]$ the kernel $H(\mathcal{K} \times \mathcal{M}(\mathcal{G})) H(\mathcal{L})^{\dagger}$ of the canonical projection

$$
H(\mathcal{K} \times \mathcal{M}(\mathcal{G})) \longrightarrow H(\mathcal{G})
$$

is a block ideal of $H(\mathcal{K} \times \mathcal{M}(\mathcal{G}))$, so that we obtain an isomorphism

$$
H(\mathcal{K} \times \mathcal{M}(\mathcal{G})) \cong H(\mathcal{K} \times \mathcal{M}(\mathcal{G})) H(\mathcal{L})^{\dagger} \oplus H(\mathcal{G})
$$

of associative algebras. Since $\mathcal{M}(\mathcal{G})$ is multiplicative, we have $H(\mathcal{M}(\mathcal{G})) \cong k^{n}$ for $n=\operatorname{ord}(\mathcal{M}(\mathcal{G}))=\operatorname{ord}(X(\mathcal{M}(\mathcal{G})))$, so that each block of $H(\mathcal{M}(\mathcal{G}))$ corresponds to a character $\lambda \in X(\mathcal{M}(\mathcal{G}))$. Thus, letting $k^{(\lambda)}$ denote the block corresponding to $\lambda$, we have

$$
h . x=\lambda(h) x \quad \forall h \in H(\mathcal{M}(\mathcal{G})), x \in k^{(\lambda)},
$$

as well as

$$
(*) \quad H(\mathcal{K} \times \mathcal{M}(\mathcal{G})) \cong H(\mathcal{K}) \otimes_{k} H(\mathcal{M}(\mathcal{G})) \cong \bigoplus_{\lambda \in X(\mathcal{M}(\mathcal{G}))} H(\mathcal{K}) \otimes_{k} k^{(\lambda)} \cong H(\mathcal{K})^{n} .
$$

In particular, each simple $(\mathcal{K} \times \mathcal{M}(\mathcal{G}))$-module is of the form $S \otimes_{k} k_{\lambda}$ for some simple $\mathcal{K}$-module $S$ and some character $\lambda \in X\left(\mathcal{M}(\mathcal{G})\right.$ ). The module $S \otimes_{k} k_{\lambda}$ belongs to $H(\mathcal{G})$ if and only if it is annihilated by $H(\mathcal{L})^{\dagger}$. By the above, this happens precisely when $\mathcal{M}(\mathcal{K})$ acts on $S$ via $\left.\lambda\right|_{\mathcal{M}(\mathcal{K})}$.

Now let $S$ be a simple $\mathcal{K}$-module. Schur's Lemma provides a character $\gamma \in$ $X(\mathcal{M}(\mathcal{K}))$ such that $H(\mathcal{M}(\mathcal{K}))$ operates on $S$ via $\gamma$. The canonical inclusion $\mathcal{M}(\mathcal{K}) \hookrightarrow \mathcal{M}(\mathcal{G})$ corresponds to the canonical restriction map

$$
\mathcal{O}(\mathcal{M}(\mathcal{G})) \stackrel{\text { res }}{\longrightarrow} \mathcal{O}(\mathcal{M}(\mathcal{K}))
$$

which is a surjective homomorphism of commutative Hopf algebras. As both groups involved are multiplicative, their function algebras coincide with the group algebras of their character groups. Accordingly, the canonical restriction map $X(\mathcal{M}(\mathcal{G})) \longrightarrow$ $X(\mathcal{M}(\mathcal{K}))$ is also surjective. We can therefore find a character $\lambda \in X(\mathcal{M}(\mathcal{G}))$ such that $\gamma=\left.\lambda\right|_{\mathcal{M}(\mathcal{K})}$. Consequently, the simple $(\mathcal{K} \times \mathcal{M}(\mathcal{G}))$-module $S \otimes_{k} k_{\lambda}$ belongs to $H(\mathcal{G})$. Let $\mathcal{B} \subset H(\mathcal{K})$ be the block of $S$. Then $S \otimes_{k} k_{\lambda}$ belongs to $\mathcal{B} \otimes_{k} k^{(\lambda)}$, proving that $\mathcal{B} \cong \mathcal{B} \otimes_{k} k^{(\lambda)}$ belongs to the block ideal $H(\mathcal{G})$.

(2) Let $\mathrm{Bl}(\mathcal{G})$ and $\mathrm{Bl}(\mathcal{K})$ be the sets of blocks of $H(\mathcal{G})$ and $H(\mathcal{K})$, respectively. In view of $(*)$ the blocks of $H(\mathcal{K} \times \mathcal{M}(\mathcal{G}))$ are of the form $\mathcal{B} \otimes_{k} k^{(\lambda)}$ for $\mathcal{B} \in \operatorname{Bl}(\mathcal{K})$ and $\lambda \in X(\mathcal{M}(\mathcal{G}))$.

For each $\gamma \in X(\mathcal{M}(\mathcal{K}))$ we denote by $\operatorname{Bl}(\mathcal{K})_{\gamma}$ the set of those blocks of $\mathcal{B}$ of $H(\mathcal{K})$ with

$$
c b=\gamma(c) b \quad \forall c \in H(\mathcal{M}(\mathcal{K})), b \in \mathcal{B} .
$$

There results a partition $\mathrm{Bl}(\mathcal{K})=\bigcup_{\gamma \in X(\mathcal{M}(\mathcal{K}))} \mathrm{Bl}(\mathcal{K})_{\gamma}$. 
Let $\mathcal{B} \in \operatorname{Bl}(\mathcal{K})_{\gamma}$. The arguments of (1) now show that the block $\mathcal{B} \otimes_{k} k^{(\lambda)}$ of $H(\mathcal{K} \times \mathcal{M}(\mathcal{G}))$ belongs to the block ideal $H(\mathcal{G})$ if and only if $\lambda \in \operatorname{res}^{-1}(\gamma)$. Accordingly, setting $\Phi(\gamma):=\operatorname{Bl}(\mathcal{K})_{\gamma} \times \operatorname{res}^{-1}(\gamma)$, we have a partition

$$
\operatorname{Bl}(\mathcal{G})=\bigcup_{\gamma \in X(\mathcal{M}(\mathcal{K}))} \bigcup_{(\mathcal{B}, \lambda) \in \Phi(\gamma)} \mathcal{B} \otimes_{k} k^{(\lambda)} .
$$

Since each fiber of the restriction map res : $X(\mathcal{M}(\mathcal{G})) \longrightarrow X(\mathcal{M}(\mathcal{K}))$ has cardinality

$$
\operatorname{ord}(\text { ker res })=\frac{\operatorname{ord}(X(\mathcal{M}(\mathcal{G})))}{\operatorname{ord}(X(\mathcal{M}(\mathcal{K})))}=\frac{\operatorname{ord}(\mathcal{M}(\mathcal{G}))}{\operatorname{ord}(\mathcal{M}(\mathcal{K}))}=\operatorname{ord}(\mathcal{M}(\mathcal{G}) / \mathcal{M}(\mathcal{K})),
$$

we obtain from the above partition that

$$
\operatorname{nb}_{\mathcal{G}}=\sum_{\gamma \in X(\mathcal{M}(\mathcal{K}))}\left|\operatorname{Bl}(\mathcal{K})_{\gamma}\right| \operatorname{ord}(\mathcal{M}(\mathcal{G}) / \mathcal{M}(\mathcal{K}))=\operatorname{ord}(\mathcal{M}(\mathcal{G}) / \mathcal{M}(\mathcal{K})) \operatorname{nb}_{\mathcal{K}}
$$

as desired.

Remark. Since the representation type of a block $\mathcal{B} \subset H(\mathcal{G})$ coincides with that of $\mathcal{B} \otimes_{k} k^{(\lambda)}$, part (2) of the foregoing result also holds for the number of blocks of a given representation type. By the same token, one has a similiar correspondence for blocks having modules of a given dimension.

An associative $k$-algebra $\Lambda$ is referred to as tame, if it is not representationfinite, and if for every $d>0$ there exist $(\Lambda, k[X])$-bimodules $M_{1}, \ldots, M_{n(d)}$ that are finitely generated free right $k[X]$-modules and such that all except finitely many indecomposable modules of dimension $d$ are isomorphic to $M_{i} \otimes_{k[X]} S$ for some simple $k[X]$-module $S$ and some $i \in\{1, \ldots, n(d)\}$.

Following [40, p. 174] we say that an algebra $\Lambda$ is special biserial if $\Lambda$ is Morita equivalent to a bound quiver algebra $k[Q] / I$ satisfying

(SB1) each vertex of $Q$ is the starting point and end point of at most two arrows, and

(SB2) for any arrow $\alpha$ of $Q$, there is at most one arrow $\beta$ and one arrow $\gamma$ with $\alpha \beta \notin I$ and $\gamma \alpha \notin I$.

For a self-injective special biserial algebra $\Lambda$, the heart $\operatorname{Rad}(P) / \operatorname{Soc}(P)$ of every indecomposable projective $\Lambda$-module $P$ is a direct sum of at most two uniserial modules [40, (5.1)].

Given $r \geq 1$, we denote by $\Delta_{r}$ the quiver with underlying set of vertices $\mathbb{Z} /\left(2 p^{r-1}\right)$ and arrows $\alpha_{i}: i \rightarrow i+1 \quad ; \quad \beta_{i}: i \rightarrow i-1 \quad$ for $i \in \mathbb{Z} /\left(2 p^{r-1}\right)$ :

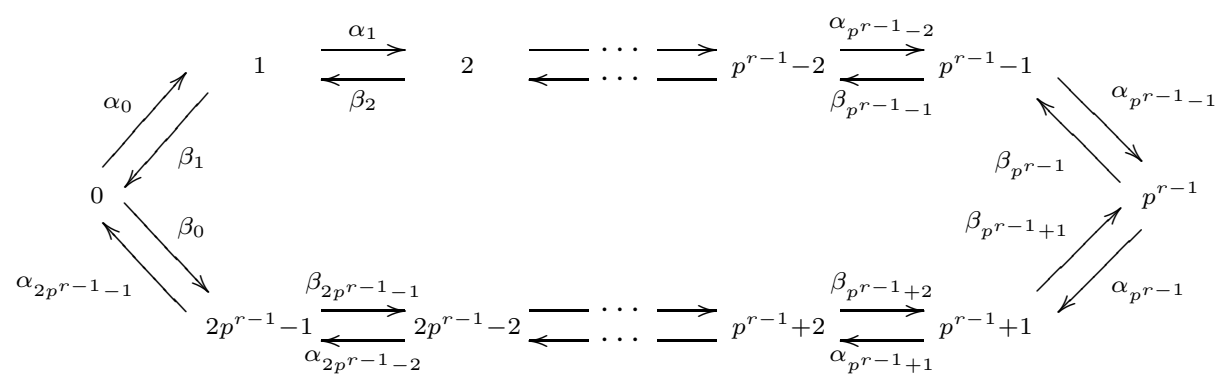


The map

$$
g: \mathbb{Z} /\left(2 p^{r-1}\right) \longrightarrow \mathbb{Z} /\left(2 p^{r-1}\right) \quad ; \quad i \mapsto i+2
$$

is an automorphism of $\Delta_{r}$ of order $p^{r-1}$, so that the subgroup $G_{r} \subset \operatorname{Aut}\left(\Delta_{r}\right)$ generated by $g$ is isomorphic to $\mathbb{Z} /\left(p^{r-1}\right)$. Note that $\Delta_{1}$ is the quiver

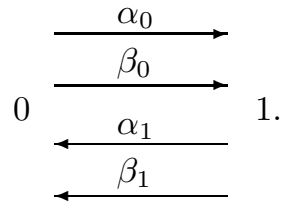

For $n \geq 0$, let $J_{r, n} \subset k\left[\Delta_{r}\right]$ be the ideal generated by

$$
\left\{\left(\beta_{i+1} \alpha_{i}\right)^{p^{n}}-\left(\alpha_{i-1} \beta_{i}\right)^{p^{n}}, \quad \alpha_{i+1} \alpha_{i}, \quad \beta_{i} \beta_{i+1} \quad ; \quad i \in \mathbb{Z} /\left(2 p^{r-1}\right)\right\} .
$$

The bound quiver algebras $\mathcal{N}^{2}(r, n)=k\left[\Delta_{r}\right] / J_{r, n}$ are special biserial. According to [18, (7.1)] the double Nakayama algebras $\mathcal{N}^{2}(r, n)$ are precisely the basic algebras of the tame principal blocks of infinitesimal group schemes.

In the following, we let $\mathfrak{n}_{n}$ be the $n$-dimensional nil-cyclic restricted Lie algebra. By definition, there exists $x \in \mathfrak{n}_{n}$ with

$$
\mathfrak{n}_{n}:=\bigoplus_{i=0}^{n-1} k x^{[p]^{i}} \quad ; \quad x^{[p]^{n}}=0 .
$$

The largest toral ideal and the largest $p$-nilpotent ideal of a restricted Lie algebra $(\mathfrak{g},[p])$ will be denoted $T(\mathfrak{g})$ and $\operatorname{rad}_{p}(\mathfrak{g})$, respectively. As before, $\{e, h, f\}$ denotes the standard basis of $\mathfrak{s l}(2)$.

Lemma 4.2. Suppose that $(\mathfrak{a},[p])$ is an abelian restricted Lie algebra such that the restricted enveloping algebra $U_{0}(\mathfrak{a})$ is representation-finite, and let $\psi: \mathfrak{s l}(2) \longrightarrow \mathfrak{a}$ be a semisimple p-semilinear form. Then the following statements hold:

(1) Each block of $U_{0}\left(\mathfrak{s l}(2)_{\psi}\right)$ is Morita equivalent to $\mathcal{N}^{2}(1, n)$ or $k[X] /\left(X^{p^{n}}\right)$, where $n:=\operatorname{dim}_{k} \operatorname{rad}_{p}(\mathfrak{a})$.

(2) If $S$ is a simple $U_{0}\left(\mathfrak{s l}(2)_{\psi}\right)$-module, then $\operatorname{dim}_{k} S \leq p$ with equality holding precisely when $S$ belongs to a representation-finite block.

Proof. Since $U_{0}(\mathfrak{a})$ has finite representation type, [13, (4.3)] provides a decomposition

$$
\mathfrak{a}=\mathfrak{n}_{n} \oplus \mathfrak{t}
$$

where $\mathfrak{t}:=T(\mathfrak{a})$ and $\mathfrak{n}_{n}=\operatorname{rad}_{p}(\mathfrak{a})$. We let $\psi_{n}$ and $\psi_{\mathfrak{t}}$ denote the two corresponding components of $\psi$. Consider the restricted Lie algebra $\mathfrak{s l}(2)_{\psi_{n}}$ as well as the $p$ semilinear map

$$
\psi_{\mathfrak{t}}^{\prime}: \mathfrak{s l}(2)_{\psi_{n}} \longrightarrow T\left(\mathfrak{s l}(2)_{\psi}\right) \quad ; \quad x+v \mapsto \psi_{\mathfrak{t}}(x) .
$$

For $\gamma \in X(\mathfrak{t})$ we define $\chi_{\gamma} \in \mathfrak{s l}(2)_{\psi_{n}}^{*}$ to be the linear form given by

$$
\chi_{\gamma}(z)^{p}=\gamma\left(\psi_{\mathfrak{t}}^{\prime}(z)\right) \quad \forall z \in \mathfrak{s l}(2)_{\psi_{n}} .
$$

Since $\mathfrak{a}=C\left(\mathfrak{s l}(2)_{\psi}\right)$, we have $T\left(\mathfrak{s l}(2)_{\psi}\right)=T(\mathfrak{a})=\mathfrak{t}$, and a consecutive application of [17, (8.1)] and [17, (8.2)] provides an isomorphism

$$
U_{0}\left(\mathfrak{s l}(2)_{\psi}\right) \cong \bigoplus_{\gamma \in X(\mathfrak{t})} U_{\chi_{\gamma}}\left(\mathfrak{s l}(2)_{\psi_{n}}\right)
$$


of $k$-algebras. Accordingly, it suffices to verify (1) and (2) for blocks and simple modules of each $U_{\chi_{\gamma}}\left(\mathfrak{s l}(2)_{\psi_{n}}\right)$.

If $\chi_{\gamma}=0$, then [17, (7.1)] implies that a block $\mathcal{B} \subset U_{0}\left(\mathfrak{s l}(2)_{\psi_{n}}\right)$ is either Morita equivalent to $\mathcal{N}^{2}(1, n)$ or to $k[X] /\left(X^{p^{n}}\right)$. We also obtain $\operatorname{dim}_{k} S \leq p$. Moreover, the algebra $U_{0}\left(\mathfrak{s l}(2)_{\psi_{n}}\right)$ possesses exactly one simple module $\mathrm{St}_{1}$ of dimension $p$ (the pull-back of the Steinberg module along the canonical projection $\mathfrak{s l}(2)_{\psi_{n}} \stackrel{\pi}{\longrightarrow} \mathfrak{s l}(2)$ ). By the remark following [17, (1.5)] the corresponding block of $U_{0}\left(\mathfrak{s l}(2)_{\psi_{n}}\right)$ is a Nakayama algebra.

Now suppose that $\chi_{\gamma} \neq 0$. According to the proof of [17, (8.10)] a block $\mathcal{B} \subset U_{\chi_{\gamma}}\left(\mathfrak{s l}(2)_{\psi_{n}}\right)$ is Morita equivalent to $k[X] /\left(X^{p^{n}}\right)$. Since $\chi_{\gamma}$ annihilates the $p$-nilpotent radical $\mathfrak{n}_{n}$ of $\mathfrak{s l}(2)_{\psi_{n}}$, we obtain a linear map $\hat{\chi}_{\gamma}: \mathfrak{s l}(2) \longrightarrow k$ such that $\hat{\chi}_{\gamma} \circ \pi=\chi_{\gamma}$. By construction, $\chi_{\gamma}$ is a semisimple linear form, and $S$ has the structure of a simple $U_{\hat{\chi}_{\gamma}}(\mathfrak{s l}(2))$-module. By virtue of [21, (2.3)] we therefore have $\operatorname{dim}_{k} S=p$.

Let $\mathcal{G}$ be an infinitesimal group and $\chi \in X(\mathcal{M}(\mathcal{G}))$ a character. We denote by $\bmod (\mathcal{G}, \chi)$ the full subcategory of $\bmod H(\mathcal{G})$ consisting of those $\mathcal{G}$-modules $M$ for which

$$
h . m=\chi(h) m \quad \forall h \in H(\mathcal{M}(\mathcal{G})), m \in M .
$$

Given $M, N \in \bmod (\mathcal{G}, \chi)$, the Hopf algebra $H(\mathcal{M}(\mathcal{G}))$ acts trivially on $\operatorname{Hom}_{\mathcal{G}_{1}}(M, N)$. As this also applies to $H\left(\mathcal{G}_{1}\right)$, it follows that every $\mathcal{G}$-module $N \in \bmod (\mathcal{G}, \chi)$ defines a functor

$$
\bmod (\mathcal{G}, \chi) \longrightarrow \bmod \mathcal{G} / \mathcal{N}(\mathcal{G}) \quad ; \quad M \mapsto \operatorname{Hom}_{\mathcal{G}_{1}}(M, N) .
$$

Since the forgetful functor $\mathcal{F}: \bmod (\mathcal{G}, \chi) \longrightarrow \bmod H\left(\mathcal{G}_{1}\right)$ is exact and sends projectives to projectives, the functors

$$
\bmod (\mathcal{G}, \chi) \longrightarrow \bmod k \quad ; \quad M \mapsto \operatorname{Ext}_{\mathcal{G}_{1}}^{n}(M, N)
$$

naturally identify with the right derived functors of the above functor and thus also map $\mathcal{G}$-modules to $\mathcal{G} / \mathcal{N}(\mathcal{G})$-modules (cf. [29, (I.6.6)]).

For each $i \geq 0$, we denote by $L(i)$ the simple SL(2)-module of highest weight $i \in \mathbb{N}_{0}$. If $i \leq p-1$, then $\left.L(i)\right|_{\mathrm{SL}(2)_{1}}$ is simple (cf. [29, (II.3.15)]). We let $L(i)^{[1]}$ be the SL(2)-module with underlying $k$-space $L(i)$ and action twisted by the Frobenius endomorphism of SL(2).

Given an infinitesimal group $\mathcal{G}$, the factor $\operatorname{group} \operatorname{Cent}(\mathcal{G}) / \mathcal{M}(\mathcal{G})$ is a unipotent group, whose length will be denoted $\ell(\operatorname{Cent}(\mathcal{G}) / \mathcal{M}(\mathcal{G}))$. We let $\mathcal{W}_{m}$ be the smooth abelian unipotent group scheme of Witt vectors of length $m$ (cf. [9, $(\mathrm{V}, \S 1,1.6)]$ ).

Recall that a module $M$ of an associative $k$-algebra $\Lambda$ is referred to as sincere if every simple $\Lambda$-module is a composition factor of $M$.

Theorem 4.3. Let $\mathcal{G}$ be an infinitesimal group with tame principal block. Suppose that $r:=\operatorname{ht}(\mathcal{G} / \mathcal{M}(\mathcal{G})) \geq 2$, and put $n:=\ell(\operatorname{Cent}(\mathcal{G}) / \mathcal{M}(\mathcal{G}))$. Let $\mathcal{B} \subset H(\mathcal{G})$ be a block.

(1) If $\mathcal{B}$ possesses a simple module of dimension $\neq p$, then $\mathcal{B}$ is Morita equivalent to $\mathcal{N}^{2}(r, n)$.

(2) If $\mathcal{B}$ affords a simple module of dimension $p$, then $\mathcal{B}$ is Morita equivalent to $k[X] /\left(X^{p^{n}}\right)$.

Proof. We set $\mathcal{N}:=\mathcal{N}(\mathcal{G})$, and let $S, S^{\prime}$ be simple $\mathcal{B}$-modules. According to Lemma 3.6 and [9, (IV, $\S 1,4.5)]$ the factor group $\mathcal{G} / \mathcal{G}_{1}$ is, as an extension of the 
multiplicative groups $\mathcal{G} / \mathcal{N}$ and $\mathcal{N} / \mathcal{G}_{1}$, multiplicative. Consequently, 29, (I.6.9)] provides an isomorphism

$$
\operatorname{Ext}_{\mathcal{G}}^{1}\left(S, S^{\prime}\right) \cong \operatorname{Ext}_{\mathcal{G}_{1}}^{1}\left(S, S^{\prime}\right)^{\mathcal{G}} .
$$

Since $S$ is a simple $\mathcal{B}$-module, Schur's Lemma provides a character $\chi \in X(\mathcal{M}(\mathcal{G}))$ such that $H(\mathcal{M}(\mathcal{G}))$ acts on $S$ via $\chi$. By general principles, we thus have $\bmod \mathcal{B} \subset$ $\bmod (\mathcal{G}, \chi)$, and by our earlier observations, the groups $\mathcal{M}(\mathcal{G})$ and $\mathcal{G}_{1}$ operate trivially on $\operatorname{Ext}_{\mathcal{G}_{1}}^{1}\left(S, S^{\prime}\right)$, so that we actually have

$$
\operatorname{Ext}_{\mathcal{G}}^{1}\left(S, S^{\prime}\right) \cong \operatorname{Ext}_{\mathcal{G}_{1}}^{1}\left(S, S^{\prime}\right)^{\mathcal{G} / \mathcal{N}} .
$$

Thus, the determination of the Gabriel quiver of $\mathcal{B}$ necessitates a closer analysis of the $\mathcal{G} / \mathcal{N}$-module $\operatorname{Ext}_{\mathcal{G}_{1}}^{1}\left(S, S^{\prime}\right)$.

By general theory [44, (9.5)], the center

$$
\operatorname{Cent}(\mathcal{G})=\mathcal{M}(\mathcal{G}) \times \mathcal{U}
$$

is a direct product of $\mathcal{M}(\mathcal{G})$, and a unipotent subgroup $\mathcal{U}$, which is necessarily normal in $\mathcal{G}$. In our situation, Theorem 1.1 ensures that $\mathcal{U}$ is contained in $\mathcal{G}_{1}$, so that $\mathcal{U}$, and thereby $\operatorname{Cent}(\mathcal{G})$, acts trivially on $\operatorname{Ext}_{\mathcal{G}_{1}}^{1}\left(S, S^{\prime}\right)$. It follows that the action of $\mathcal{G}$ on $\operatorname{Ext}_{\mathcal{G}_{1}}^{1}\left(S, S^{\prime}\right)$ factors through to $\mathcal{G} / \operatorname{Cent}(\mathcal{G})$.

Thanks to Proposition 3.5 there exist a quotient map $\pi: \mathcal{G} \longrightarrow \mathcal{Q}_{[r]}$ with kernel $\operatorname{Cent}(\mathcal{G})$ and a multiplicative subgroup $\mathcal{M} \subset \mathcal{G}$ such that $\left.\pi\right|_{\mathcal{M}}: \mathcal{M} \longrightarrow T_{r}$ is a quotient map with kernel $\mathcal{M}(\mathcal{G})$ and $\mathcal{G}=\mathcal{G}_{1} \mathcal{M}$. Let $\mathfrak{g}:=\operatorname{Lie}(\mathcal{G})$ be the Lie algebra of $\mathcal{G}$. According to Lemma 3.4 we can find a $p$-semilinear map $\psi: \mathfrak{s l}(2) \longrightarrow C(\mathfrak{g})$ such that $\mathfrak{g} \cong \mathfrak{s l}(2)_{\psi}$. In fact, the isomorphism is induced by the differential $d \pi$ : $\mathfrak{g} \longrightarrow \mathfrak{s l}(2)$ of $\pi$. Decomposing $C(\mathfrak{g})$ into its $p$-nilpotent and toral part, we write

$$
C(\mathfrak{g})=\mathfrak{n}_{n} \oplus \mathfrak{t}
$$

as well as $\psi=\psi_{n}+\psi_{\mathfrak{t}}$. Owing to Lemma 2.2 and [17, (8.1)] the canonical inclusion $\mathfrak{s l}(2)_{\psi_{n}} \hookrightarrow \mathfrak{s l}(2)_{\psi}$ induces a $T$-equivariant isomorphism

$$
U_{0}\left(\mathfrak{s l}(2)_{\psi}\right) \cong \bigoplus_{\gamma \in X(\mathfrak{t})} U_{\chi_{\gamma}}\left(\mathfrak{s l}(2)_{\psi_{n}}\right)
$$

of $k$-algebras.

By our observations above, the quotient map $\pi$ endows the space $\operatorname{Ext}_{\mathcal{G}_{1}}^{1}(S, N)$ with the structure of a $\mathcal{Q}_{[r]}$-module for any $\mathcal{B}$-module $N$. Composing $\pi$ with the canonical map $\mathcal{Q}_{[r]} \longrightarrow \mathcal{Q}_{[r]} / \mathrm{SL}(2)_{1} \cong T_{r} / T_{1}$ we obtain a quotient map $\mathcal{G} / \mathcal{N} \longrightarrow$ $T_{r} / T_{1}$. In view of Lemma 3.6 both groups involved have the same order, so that the above map is an isomorphism. Consequently, the subgroup $T_{1} \subset T_{r}$ operates trivially on $\operatorname{Ext}_{\mathcal{G}_{1}}^{1}(S, N)$.

By virtue of Theorem $3.7(1)$ the restriction $\left.S\right|_{\mathcal{G}_{1}}$ of every simple $\mathcal{G}$-module $S$ is simple. In view of $(*)$ the restrictions of the simple $\mathcal{B}$-modules all belong to one block of the Hopf algebra $H\left(\mathcal{G}_{1}\right)$. Thus, the differential of the representation of $S$ makes $S$ a simple $U_{0}(\mathfrak{g})$-module, and all $\mathcal{B}$-modules belong to the same block of $U_{0}(\mathfrak{g})$. Owing to $(* *)$ there exists a character $\gamma \in X(\mathfrak{t})$ such that the restriction $\left.M\right|_{\mathfrak{s l}(2)_{\psi_{n}}}$ of the differential of a $\mathcal{B}$-module $M$ is a $U_{\chi_{\gamma}}\left(\mathfrak{s l}(2)_{\psi_{n}}\right)$-module.

(1) Now let $\mathcal{B} \subset H(\mathcal{G})$ be a block affording a simple module $S$ of dimension $\operatorname{dim}_{k} S=i+1<p$. We shall proceed in several steps.

(a) The block $\mathcal{B}$ has Gabriel quiver $\Delta_{r}$. 
Since $\psi$ is semisimple, the $p$-character $\chi_{\gamma}$ induces a semisimple $p$-character $\hat{\chi}_{\gamma} \in$ $\mathfrak{s l}(2)^{*}$ such that $S$ is a $U_{\hat{\chi}_{\gamma}}(\mathfrak{s l}(2))$-module. Thanks to [21, (2.2)] we have $\hat{\chi}_{\gamma}=0=$ $\chi_{\gamma}$, so that $S$ is a simple $U_{0}(\mathfrak{s l}(2))$-module. Thus, if $S^{\prime}$ is another simple $\mathcal{B}$-module, then, by Lemma 2.3 and [17, (1.3)], we have isomorphisms

$$
\operatorname{Ext}_{\mathcal{G}_{1}}^{1}\left(S, S^{\prime}\right) \cong \operatorname{Ext}_{U_{0}(\mathfrak{g})}^{1}\left(S, S^{\prime}\right) \cong \operatorname{Ext}_{U_{0}\left(\mathfrak{s l}(2)_{\psi_{n}}\right)}^{1}\left(S, S^{\prime}\right) \cong \operatorname{Ext}_{U_{0}(\mathfrak{s l}(2))}^{1}\left(S, S^{\prime}\right)
$$

of $T_{r}$-modules.

According to Lemma 2.1 the simple $U_{0}(\mathfrak{s l}(2))$-modules $S$ and $S^{\prime}$ are also $\left(U_{0}(\mathfrak{s l}(2)), T_{r}\right)$-modules. Since the $\mathfrak{s l}(2)$-module $S$ is isomorphic to $L(i)$, the $H\left(T_{r}\right)$ moduleHom $_{U_{0}(\mathfrak{s l}(2))}(L(i), S)$ is one dimensional and annihilated by $H\left(T_{1}\right)^{\dagger}$. The arguments of Proposition 3.2 now provide a character $\lambda \in X\left(T_{r} / T_{1}\right)$ such that $S \cong L(i) \otimes_{k} k_{\lambda}$. By the same token, we have $S^{\prime} \cong L(j) \otimes_{k} k_{\mu}$ for some $\mu \in X\left(T_{r} / T_{1}\right)$. Consequently, the arguments of [20, (5.3)] in conjunction with the main theorem of 34] now establish the following isomorphisms of $T_{r}$-modules:

$$
\operatorname{Ext}_{\mathcal{G}_{1}}^{1}\left(S, S^{\prime}\right) \cong\left\{\begin{array}{cc}
L(1)^{[1]} \otimes_{k} k_{\mu-\lambda} & i+j=p-2 \\
0 & \text { otherwise }
\end{array}\right.
$$

Recall that $T_{r}$ acts on the standard SL(2)-module $L(1)$ via two characters $\alpha,-\alpha \in$ $X\left(T_{r}\right) \cong \mathbb{Z} /\left(p^{r}\right)$, both having order $p^{r}$. Hence $T_{r}$ operates on the Frobenius twist $L(1)^{[1]}$ via $\beta:=p \alpha$ and $-\beta$, which are generators of $X\left(T_{r} / T_{1}\right) \cong \mathbb{Z} /\left(p^{r-1}\right)$.

Owing to $(*)$ we have isomorphisms

$$
(* * *) \quad \operatorname{Ext}_{\mathcal{G}}^{1}\left(S, S^{\prime}\right) \cong \operatorname{Ext}_{\mathcal{G}_{1}}^{1}\left(S, S^{\prime}\right)^{\mathcal{G} / \mathcal{N}} \cong\left(L(1)^{[1]} \otimes_{k} k_{\mu-\lambda}\right)^{T_{r}}
$$

whenever $\operatorname{dim}_{k} S+\operatorname{dim}_{k} S^{\prime}=p$. Thus, the extension group is non-trivial if and only if $\mu-\lambda \in\{\beta,-\beta\}$. This readily implies that the block $\mathcal{B}$ has Gabriel quiver $\Delta_{r}$.

(b) The block $\mathcal{B}$ is an $X(\mathcal{G} / \mathcal{N})$-submodule of $H(\mathcal{G})$.

From $(* * *)$ we see that $S \otimes_{k} k_{\lambda}$ belongs to $\mathcal{B}$ for every character $\lambda \in X(\mathcal{G} / \mathcal{N}) \cong$ $\mathbb{Z} /\left(p^{r-1}\right)$. As $X(\mathcal{G} / \mathcal{N})$ permutes the central idempotents of $H(\mathcal{G})$, it follows that the idempotent definining $\mathcal{B}$ is a fixed-point of $X(\mathcal{G} / \mathcal{N})$.

(c) $\mathcal{B}^{X(\mathcal{G} / \mathcal{N})}$ is a block of $H(\mathcal{N})$.

Since the group $\mathcal{G} / \mathcal{N}$ is multiplicative, [29, (I.6.9)] implies

$$
\operatorname{Ext}_{\mathcal{G}}^{1}\left(S, S^{\prime}\right) \cong \operatorname{Ext}_{\mathcal{N}}^{1}\left(S, S^{\prime}\right)^{\mathcal{G} / \mathcal{N}}
$$

for any two simple $\mathcal{G}$-modules $S$ and $S^{\prime}$. According to Theorem 3.7(1) the restrictions to $H(\mathcal{N})$ of the simple $\mathcal{B}$-modules are simple, so that our formula implies that all these belong to one block of $H(\mathcal{N})$.

Let $e_{\mathcal{B}}$ be the primitive central idempotent of $H(\mathcal{G})$ defining the block $\mathcal{B}$. Since $X(\mathcal{G} / \mathcal{N})$ permutes the central idempotents, part (b) and Theorem 3.7(2) imply $e_{\mathcal{B}} \in H(\mathcal{G})^{X(\mathcal{G} / \mathcal{N})}=H(\mathcal{N})$. Consequently, we have

$$
H(\mathcal{N}) e_{\mathcal{B}} \subset \mathcal{B}^{X(\mathcal{G} / \mathcal{N})} .
$$

By the arguments of [20, (5.1)] the group $X(\mathcal{G} / \mathcal{N})$ acts transitively on those fibers of the canonical restriction functor $\bmod H(\mathcal{G}) \longrightarrow \bmod H(\mathcal{N})$ that are defined by simple $\mathcal{N}$-modules. Applying Theorem 3.7 and Lemma3.3 successively, we conclude that every simple $\mathcal{N}$-module is the restriction of a simple $\mathcal{G}$-module.

Let $V$ be a simple $H(\mathcal{N})$-module belonging to the block ideal $H(\mathcal{N}) e_{\mathcal{B}}$. Since $V$ has the structure of a $\mathcal{G}$-module that extends its $\mathcal{N}$-structure, $V=\left.e_{\mathcal{B}} \cdot V\right|_{\mathcal{N}}=e_{\mathcal{B}} \cdot V$, so that $V$ belongs to $\mathcal{B}$. As a result, the restriction functor induces a surjection 
$\operatorname{Irr}(\mathcal{B}) \longrightarrow \operatorname{Irr}\left(H(\mathcal{N}) e_{\mathcal{B}}\right)$ between the sets of isoclasses of simple $\mathcal{B}$-modules and simple $H(\mathcal{N}) e_{\mathcal{B}}$-modules. By virtue of $(\dagger)$ it now follows that the Gabriel quiver of $H(\mathcal{N}) e_{\mathcal{B}}$ is connected. As a result, the block ideal $H(\mathcal{N}) e_{\mathcal{B}}$ is in fact a block of $H(\mathcal{N})$.

In view of Lemma 4.1 the simple $\mathcal{N}$-modules are just the simple $\mathcal{G}_{1}$-modules, and the blocks of $H(\mathcal{N})$ occur in $H\left(\mathcal{G}_{1}\right)$. A consecutive application of Lemmas 3.4 and 4.2 now shows that the dimensions of the simple $\mathcal{N}$-modules (and thereby the dimensions of the simple $\mathcal{G}$-modules) are bounded by $p$. By the same token, the simple $\mathcal{N}$-modules belonging to a block having a simple module of dimension $<p$ all are of dimension $<p$.

Now let $\mathcal{B}_{1}, \ldots, \mathcal{B}_{\ell}$ be the blocks of $H(\mathcal{G})$ containing a module of dimension $<p$. Then every simple $\mathcal{B}_{i}$-module has dimension $<p$, and the remaining blocks of $H(\mathcal{G})$ form an $X(\mathcal{G} / \mathcal{N})$-invariant block ideal $\mathcal{C}=H(\mathcal{G}) e_{\mathcal{C}}$, defined by a central idempotent $e_{\mathcal{C}} \in H(\mathcal{G})^{X(\mathcal{G} / \mathcal{N})}=H(\mathcal{N})$. Since every simple $H(\mathcal{N})$-module of dimension $p$ is the restriction of a simple $\mathcal{C}$-module, such a module belongs to $H(\mathcal{C}) e_{\mathcal{C}}$. Consequently, $H(\mathcal{N})=\bigoplus_{i=1}^{\ell} H(\mathcal{N}) e_{\mathcal{B}_{i}} \oplus H(\mathcal{N}) e_{\mathcal{C}}$, so that

$$
H(\mathcal{N})=\bigoplus_{i=1}^{\ell} H(\mathcal{N}) e_{\mathcal{B}_{i}} \oplus H(\mathcal{N}) e_{\mathcal{C}} \subset \bigoplus_{i=1}^{\ell} \mathcal{B}_{i}^{X(\mathcal{G} / \mathcal{N})} \oplus \mathcal{C}^{X(\mathcal{G} / \mathcal{N})}=H(\mathcal{N})
$$

In particular, $\mathcal{B}_{i}^{X(\mathcal{G} / \mathcal{N})}=H(\mathcal{N}) e_{\mathcal{B}_{i}}$ is a block of $H(\mathcal{N})$ for every $i \in\{1, \ldots, \ell\}$.

(d) Let $P$ be a principal indecomposable $\mathcal{B}$-module. Then $P$ is not sincere.

According to Theorem 1.1 there exists a natural number $m \in \mathbb{N}_{0}$ such that $\operatorname{Cent}(\mathcal{G}) \cong\left(\mathcal{W}_{m}\right)_{1} \times \mathcal{M}(\mathcal{G})$. Thus, $\left(\mathcal{W}_{m}\right)_{1} \cong \operatorname{Cent}(\mathcal{G}) / \mathcal{M}(\mathcal{G})$, so that

$$
m=\ell(\operatorname{Cent}(\mathcal{G}) / \mathcal{M}(\mathcal{G}))=n .
$$

Since $H\left(\left(\mathcal{W}_{n}\right)_{1}\right) \cong k[X] /\left(X^{p^{n}}\right)$ there exists an element $v_{0} \in H\left(\left(\mathcal{W}_{n}\right)_{1}\right)^{\dagger}$ such that $H\left(\left(\mathcal{W}_{n}\right)_{1}\right)=k\left[v_{0}\right]$. Thus, $v_{0}$ is a nilpotent element of the center of $H(\mathcal{G})$ that satisfies $v_{0}^{p^{n}}=0$. Setting $I:=H(\mathcal{G}) v_{0}$ we have $I=H(\mathcal{G}) H\left(\left(\mathcal{W}_{n}\right)_{1}\right)^{\dagger}$ as well as $H(\mathcal{G}) / I \cong H(\hat{\mathcal{G}})$, where $\hat{\mathcal{G}}=\mathcal{G} /\left(\mathcal{W}_{n}\right)_{1}$. Since $\hat{\mathcal{G}}$ is not supersolvable, [20, (1.1)] implies that the principal block $\mathcal{B}_{0}(\hat{\mathcal{G}})$ is tame. Consequently, Theorem 1.1 now provides an isomorphism $\operatorname{Cent}(\hat{\mathcal{G}}) \cong \mathcal{M}(\mathcal{G})$.

Since $I$ is nilpotent, the $\hat{\mathcal{G}}$-module $P / v_{0} P$ is projective indecomposable. Moreover, left multiplication by $v_{0}^{i}$ induces a surjection

$$
P / v_{0} P \longrightarrow v_{0}^{i} P / v_{0}^{i+1} P
$$

of $H(\hat{\mathcal{G}})$-modules for every $i \in\left\{0, \ldots, p^{n}-1\right\}$. Since $k\left[v_{0}\right]=H\left(\left(\mathcal{W}_{n}\right)_{1}\right)$ is a Hopf subalgebra of $H(\mathcal{G})$, [33, (2.6)] guarantees that $\left.P\right|_{k\left[v_{0}\right]}$ is projective. As $k\left[v_{0}\right]$ is local, the module $\left.P\right|_{k\left[v_{0}\right]}$ is free and there exists $\ell \geq 1$ with $\left.P\right|_{k\left[v_{0}\right]} \cong k\left[v_{0}\right]^{\ell}$. This readily implies $\operatorname{dim}_{k} v_{0}^{i} P / v_{0}^{i+1} P=\ell$ for every $i \in\left\{0, \ldots, p^{n}-1\right\}$, showing that the above surjections are in fact isomorphisms

$$
P / v_{0} P \cong v_{0}^{i} P / v_{0}^{i+1} P
$$

We let $\hat{\mathfrak{g}}=\operatorname{Lie}(\hat{\mathcal{G}})$ be the Lie algebra of $\hat{\mathcal{G}}$. Since the unipotent part of the center of $\hat{\mathcal{G}}$ is trivial, $(* *)$ specializes to

$$
U_{0}(\hat{\mathfrak{g}}) \cong \bigoplus_{\gamma \in X(\mathfrak{t})} U_{\chi_{\gamma}}(\mathfrak{s l}(2))
$$


Thanks to Corollary 3.8 and Lemma 3.3 the module $\left.\left(P / v_{0} P\right)\right|_{\hat{\mathcal{G}}_{1}}$ has length $\ell\left(P / v_{0} P\right)$. Since the dimension of the top of $P$ is bounded by $p-1$, the $U_{0}(\hat{\mathfrak{g}})$ module $\left.\left(P / v_{0} P\right)\right|_{\hat{\mathcal{G}}_{1}}$ affords $p$-character $\chi_{\gamma}=0$ and thus has length 4 . In view of (†) the module $P$ has at most 4 distinct composition factors. As $p \geq 3$ and $r \geq 2$ we have $4<|\operatorname{Irr}(\mathcal{B})|$, so that $P$ is not sincere.

Thanks to (a) the block $\mathcal{B}$ has Gabriel quiver $\Delta_{r}$. A consecutive application of Theorem 3.7, (b) and 2, (5.1)] ensures that $\mathcal{B}: \mathcal{B}^{X(\mathcal{G} / \mathcal{N})}$ is a Galois extension with Galois group $X(\mathcal{G} / \mathcal{N})$ acting freely on $\operatorname{Irr}(\mathcal{B})$. Owing to (c) the algebra $\mathcal{B}^{X(\mathcal{G} / \mathcal{N})}$ is a block of $H(\mathcal{N})$, which by Lemmas 4.1 and 4.2, is Morita equivalent to $\mathcal{N}^{2}(1, m)$ for $m:=\operatorname{dim}_{k} \operatorname{rad}_{p}(C(\mathfrak{g}))$. By virtue of Theorem 1.1] we have $C(\mathfrak{g})=\operatorname{Lie}(\operatorname{Cent}(\mathcal{G}))$, whence

$$
\operatorname{rad}_{p}(C(\mathfrak{g}))=\operatorname{rad}_{p}(\operatorname{Lie}(\operatorname{Cent}(\mathcal{G}))) \cong \mathfrak{n}_{n} .
$$

Thus, $m=n$, and the claim of (1) now follows by applying [18, (4.4)] and (d) successively.

(2) Now suppose that $\mathcal{B} \subset H(\mathcal{G})$ is a block having a simple module $S$ with $\operatorname{dim}_{k} S=p$. By Theorem $3.7(1)$ the restriction $\left.S\right|_{\mathcal{G}_{1}}$ is simple and (**) shows that $\left.S\right|_{\mathcal{G}_{1}}$ belongs to a block of $U_{\chi_{\gamma}}\left(\mathfrak{s l}(2)_{\psi_{n}}\right)$. If $\chi_{\gamma}=0$, then, arguing as in (a) above, we obtain $\lambda \in X\left(T_{r} / T_{1}\right)$ such that

$$
S \cong L(p-1) \otimes_{k} k_{\lambda}
$$

as a $\left(U_{0}(\mathfrak{s l}(2)), T_{r}\right)$-module. If $S^{\prime}=L(p-1) \otimes_{k} k_{\mu}$ is another $\mathcal{B}$-module, then the remark following [17, (1.3)] provides isomorphisms

$$
\operatorname{Ext}_{\mathcal{G}_{1}}^{1}\left(S, S^{\prime}\right) \cong \operatorname{Ext}_{U_{0}(\mathfrak{s l}(2))}^{1}\left(S, S^{\prime}\right) \cong \operatorname{Hom}_{U_{0}(\mathfrak{s l}(2))}(L(p-1), L(p-1)) \otimes_{k} k_{\mu-\lambda}
$$

of $T_{r}$-modules. Since $T_{r}$ operates trivially on $\operatorname{Hom}_{U_{0}(\mathfrak{s l}(2))}(L(p-1), L(p-1)) \cong$ $\operatorname{Hom}_{\mathrm{SL}(2)}(L(p-1), L(p-1))$, we obtain from $(*)$

$$
\operatorname{dim}_{k} \operatorname{Ext}_{\mathcal{G}}^{1}\left(S, S^{\prime}\right)=\operatorname{dim}_{k}\left(k_{\mu-\lambda}\right)^{T_{r}}=\delta_{\mu, \lambda} .
$$

Thus, the block $\mathcal{B}$ is a primary Nakayama algebra.

It remains to treat the case where $\chi_{\gamma} \neq 0$. As $\chi_{\gamma}$ annihilates the $p$-nilpotent radical $\mathfrak{n}_{n}$ of $\mathfrak{s l}(2)_{\psi_{n}}$, it induces a linear form $\hat{\chi}_{\gamma} \in \mathfrak{s l}(2)^{*}$. Given $S$ and $S^{\prime}$ as before, we consider the $U_{0}\left(\mathfrak{s l}(2)_{\psi_{n}}\right)$-module $M:=\operatorname{Hom}_{k}\left(S, S^{\prime}\right)$. Since $M$ is annihilated by $U_{0}\left(\mathfrak{n}_{n}\right)^{\dagger}$, the cohomology five term sequence associated to the spectral sequence $H^{m}\left(U_{0}(\mathfrak{s l}(2)), H^{\ell}\left(U_{0}\left(\mathfrak{n}_{n}\right), M\right)\right) \Rightarrow H^{m+\ell}\left(U_{0}\left(\mathfrak{s l}(2)_{\psi_{n}}\right), M\right)$ in conjunction with 21, (5.1)] provides an exact sequence

$$
\begin{aligned}
(0) \longrightarrow \operatorname{Ext}_{U_{\hat{\chi}_{\gamma}}(\mathfrak{s l}(2))}^{1}\left(S, S^{\prime}\right) & \longrightarrow \operatorname{Ext}_{U_{\chi_{\gamma}\left(\mathfrak{s l}(2)_{\psi_{n}}\right)}^{1}\left(S, S^{\prime}\right) \longrightarrow H^{1}\left(U_{0}\left(\mathfrak{n}_{n}\right), M\right)^{\mathfrak{s l}(2)_{\psi_{n}}}} \\
& \longrightarrow \operatorname{Ext}_{U_{\hat{\chi}_{\gamma}(\mathfrak{s l}(2))}\left(S, S^{\prime}\right)}^{2^{\prime}}
\end{aligned}
$$

of $T_{r}$-modules. According to [21, (2.2)] the reduced enveloping algebra $U_{\hat{\chi}_{\gamma}}(\mathfrak{s l}(2))$ is semisimple, and the above sequence yields an isomorphism

$$
\operatorname{Ext}_{U_{\chi \gamma}\left(\mathfrak{s l}(2)_{\psi_{n}}\right)}^{1}\left(S, S^{\prime}\right) \cong H^{1}\left(U_{0}\left(\mathfrak{n}_{n}\right), M\right)^{\mathfrak{s l}(2) \psi_{n}}
$$

of $T_{r}$-modules. Since $\mathfrak{n}_{n} \subset C\left(\mathfrak{s l}(2)_{\psi_{n}}\right)$ is nil-cyclic and acts trivially on $M$, we have

$$
\begin{aligned}
& H^{1}\left(U_{0}\left(\mathfrak{n}_{n}\right), M\right)^{\mathfrak{s l}(2)_{\psi_{n}}} \cong\left(H^{1}\left(U_{0}\left(\mathfrak{n}_{n}\right), k\right) \otimes_{k} M\right)^{\mathfrak{s l}(2)_{\psi_{n}}} \\
& \cong M^{\mathfrak{s l}(2)_{\psi_{n}}} \cong \operatorname{Hom}_{U_{\chi_{\gamma}}\left(\mathfrak{s l}(2)_{\psi_{n}}\right)}\left(S, S^{\prime}\right) \text {. }
\end{aligned}
$$


By virtue of [21, (2.3)] the $U_{\chi_{\gamma}}\left(\mathfrak{s l}(2)_{\psi_{n}}\right)$-module $S$ is the pullback of a baby Verma module

$$
Z_{\hat{\chi}_{\gamma}}(\lambda):=U_{\hat{\chi}_{\gamma}}(\mathfrak{s l}(2)) \otimes_{U_{\left.\hat{\chi}_{\gamma}\right|_{\mathfrak{b}}}(\mathfrak{b})} k_{\lambda}
$$

along the canonical map $U_{\hat{\chi}_{\gamma}}\left(\mathfrak{s l}(2) \psi_{n}\right) \longrightarrow U_{\hat{\chi}_{\gamma}}(\mathfrak{s l}(2))$. Here $\mathfrak{b}:=k h \oplus k e$ denotes the standard Borel subalgebra of upper triangular matrices. The canonical action of $T$ on $U_{\hat{\chi}_{\gamma}}(\mathfrak{s l}(2))$ endows $Z_{\hat{\chi}_{\gamma}}(\lambda)$ with the structure of a $\left(U_{\hat{\chi}_{\gamma}}(\mathfrak{s l}(2)) \sharp H\left(T_{r}\right)\right)$-module. Arguing as before, we can find characters $\mu, \mu^{\prime} \in X\left(T_{r} / T_{1}\right)$ such that

$$
S \cong Z_{\hat{\chi}_{\gamma}}(\lambda) \otimes_{k} k_{\mu} \quad ; \quad S^{\prime} \cong Z_{\hat{\chi}_{\gamma}}\left(\lambda^{\prime}\right) \otimes_{k} k_{\mu^{\prime}}
$$

as $\left(U_{\hat{\chi}_{\gamma}}(\mathfrak{s l}(2)) \sharp H\left(T_{r}\right)\right)$-modules. In view of [21, (2.3)] we have

$$
\begin{aligned}
\operatorname{dim}_{k} \operatorname{Hom}_{U_{\hat{\chi}_{\gamma}}(\mathfrak{s l}(2))}\left(Z_{\hat{\chi}_{\gamma}}(\lambda), Z_{\hat{\chi}_{\gamma}}\left(\lambda^{\prime}\right)\right) \\
\quad=\delta_{\lambda, \lambda^{\prime}}=\operatorname{dim}_{k} \operatorname{Hom}_{U_{\hat{\chi}_{\gamma}}(\mathfrak{s l}(2)) \sharp H\left(T_{r}\right)}\left(Z_{\hat{\chi}_{\gamma}}(\lambda), Z_{\hat{\chi}_{\gamma}}\left(\lambda^{\prime}\right)\right) .
\end{aligned}
$$

In particular, $T_{r}$ operates trivially on $\operatorname{Hom}_{U_{\hat{\chi}_{\gamma}}(\mathfrak{s l}(2))}\left(Z_{\hat{\chi}_{\gamma}}(\lambda), Z_{\hat{\chi}_{\gamma}}\left(\lambda^{\prime}\right)\right)$, and $(*)$ implies

$$
\operatorname{dim}_{k} \operatorname{Ext}_{\mathcal{G}}^{1}\left(S, S^{\prime}\right)=\delta_{\lambda, \lambda^{\prime}} \delta_{\mu, \mu^{\prime}}
$$

Thus, the block $\mathcal{B}$ is again a primary Nakayama algebra.

In either case, the Morita type of $\mathcal{B}$ may now be determined by computing the length $\ell$ of the principal indecomposable $\mathcal{B}$-module $P$. Owing to Corollary 3.8 and Lemma 3.3 we have $\ell=\ell\left(P \mid \mathcal{G}_{1}\right)$, and Lemma 4.2 now implies

$$
\ell=\operatorname{dim}_{k} \operatorname{rad}_{p}(C(\mathfrak{g}))=n,
$$

as desired.

Remark. Note that the proof also provides the dimensions of the simple $\mathcal{G}$-modules as well as their distribution over the blocks.

In order to obtain more detailed information concerning the distribution of blocks with regard to their representation type, we define an invariant for certain central extensions of Lie algebras. Let $(\mathfrak{g},[p])$ be a restricted Lie algebra, and $\psi: \mathfrak{g} \longrightarrow \mathfrak{a}$ a $p$-semilinear map to an abelian restricted Lie algebra $(\mathfrak{a},[p])$. We decompose $\mathfrak{a}$ into its $p$-nilpotent and toral part and write

$$
\mathfrak{a}=\operatorname{rad}_{p}(\mathfrak{a}) \oplus T(\mathfrak{a}) .
$$

As before, the composition of $\psi$ with the canonical projection onto the toral radical $T(\mathfrak{a})$ will be denoted $\psi_{T(\mathfrak{a})}$. Since $X(T(\mathfrak{a}))$ is an $\mathbb{F}_{p}$-vector space of dimension $\operatorname{dim}_{k} T(\mathfrak{a})$, the number

$$
\ell_{\psi}:=\left|\left\{\gamma \in X(T(\mathfrak{a})) ; \gamma \circ \psi_{T(\mathfrak{a})}=0\right\}\right|
$$

is a power of $p$. If $\mathfrak{g}$ is a centerless and perfect Lie algebra, i.e., if $C(\mathfrak{g})=(0)$ and $\mathfrak{g}=[\mathfrak{g}, \mathfrak{g}]$, then $\ell_{\psi}$ is an invariant of the isomorphism type of $\mathfrak{g}_{\psi}$ (cf. [18, (6.1)]).

Now let $\mathcal{G}$ be an infinitesimal group with tame principal block. By (1.2) and 20, (6.4)], the Lie algebra $\mathfrak{g}:=\operatorname{Lie}(\mathcal{G})$ is isomorphic to $\mathfrak{s l}(2)_{\psi}$ for a suitable $p$-semilinear map $\psi: \mathfrak{s l}(2) \longrightarrow C(\mathfrak{g})$. As $p \geq 3$, the Lie algebra $\mathfrak{s l}(2)$ is centerless and perfect. We thus define

$$
\ell_{\mathcal{G}}:=\ell_{\psi}
$$

and note that $\ell_{\mathcal{G}}$ only depends on the isomorphism type of $\mathcal{G}$. 
Corollary 4.4. Let $\mathcal{G}$ be an infinitesimal group with tame principal block. If $r=$ $\operatorname{ht}(\mathcal{G} / \mathcal{M}(\mathcal{G})) \geq 2$, and $n=\ell(\operatorname{Cent}(\mathcal{G}) / \mathcal{M}(\mathcal{G}))$, then the following statements hold:

(1) $H(\mathcal{G})$ has $\operatorname{ord}\left(\mathcal{M}(\mathcal{G}) / \mathcal{M}\left(\mathcal{G}_{1}\right)\right) \ell_{\mathcal{G}} \frac{p-1}{2}$ blocks of Morita type $\mathcal{N}^{2}(r, n)$.

(2) $H(\mathcal{G})$ has $\operatorname{ord}\left(\mathcal{G} / \mathcal{G}_{1}\right)\left(\ell_{\mathcal{G}}+p\left(\operatorname{ord}\left(\mathcal{M}\left(\mathcal{G}_{1}\right)\right)-\ell_{\mathcal{G}}\right)\right)$ blocks of Morita type $k[X] /\left(X^{p^{n}}\right)$.

Proof. According to Theorem 1.1 there exists $m \geq 0$ such that

$$
\operatorname{Cent}(\mathcal{G}) \cong \mathcal{M}(\mathcal{G}) \times\left(\mathcal{W}_{m}\right)_{1}
$$

In particular, we have $\operatorname{Cent}(\mathcal{G}) / \mathcal{M}(\mathcal{G}) \cong\left(\mathcal{W}_{m}\right)_{1}$, so that $m=n$.

Let $\mathfrak{g}:=\operatorname{Lie}(\mathcal{G})$ be the Lie algebra of $\mathcal{G}$. Another application of Theorem 1.1 implies $C(\mathfrak{g})=\operatorname{Lie}(\operatorname{Cent}(\mathcal{G})) \cong \mathfrak{n}_{n} \oplus T(\mathfrak{g})$. According to Lemma 3.4 there exists a semisimple $p$-semilinear map $\psi: \mathfrak{s l}(2) \longrightarrow C(\mathfrak{g})$ such that $\mathfrak{g} \cong \mathfrak{s l}(2)_{\psi}$. As before, we consider the decomposition $\psi=\psi_{n}+\psi_{T(\mathfrak{g})}$. Then we have

$$
U_{0}(\mathfrak{g}) \cong \bigoplus_{\gamma \in X(T(\mathfrak{g}))} U_{\chi_{\gamma}}\left(\mathfrak{s l}(2)_{\psi_{n}}\right) .
$$

If $\chi_{\gamma}=0$, then [18, (1.4)] implies that $U_{\chi_{\gamma}}\left(\mathfrak{s l}(2)_{\psi_{n}}\right)$ has $\frac{p-1}{2}$ blocks of Morita type $\mathcal{N}^{2}(1, n)$ and one block of type $k[X] /\left(X^{p^{n}}\right)$. Alternatively, [21, (2.2)] and [17, (8.10)] yield that $U_{\chi_{\gamma}}\left(\mathfrak{s l}(2)_{\psi_{n}}\right)$ has $p$ blocks of the latter type. Counting occurrences of these cases, we obtain that $H\left(\mathcal{G}_{1}\right) \cong U_{0}(\mathfrak{g})$ has $\ell_{\mathcal{G}} \frac{p-1}{2}$ blocks of type $\mathcal{N}^{2}(1, n)$ and $\ell_{\mathcal{G}}+p\left(\operatorname{ord}\left(\mathcal{M}\left(\mathcal{G}_{1}\right)\right)-\ell_{\mathcal{G}}\right)$ blocks of Morita type $k[X] /\left(X^{p^{n}}\right)$.

Since $\mathcal{N}(\mathcal{G})$ is a normal subgroup of $\mathcal{G}$ containing $\mathcal{M}(\mathcal{G})$, we have $\mathcal{M}(\mathcal{N}(\mathcal{G}))=$ $\mathcal{M}(\mathcal{G})$. Thus, the group $\mathcal{N}(\mathcal{G})$ satisfies the conditions of Lemma 4.1, so that the corresponding types of blocks occur in the Hopf algebra $H(\mathcal{N}(\mathcal{G})$ ) with $\operatorname{ord}\left(\mathcal{M}(\mathcal{G}) / \mathcal{M}\left(\mathcal{G}_{1}\right)\right)$-fold multiplicities. Observing Lemma 3.6 we have

$$
\begin{aligned}
p^{r-1} \operatorname{ord}\left(\mathcal{M}(\mathcal{G}) / \mathcal{M}\left(\mathcal{G}_{1}\right)\right) & =\frac{\operatorname{ord}(\mathcal{G})}{\operatorname{ord}\left(\mathcal{G}_{1} \mathcal{M}(\mathcal{G})\right)} \frac{\operatorname{ord}(\mathcal{M}(\mathcal{G}))}{\operatorname{ord}\left(\mathcal{M}\left(\mathcal{G}_{1}\right)\right)} \\
& =\frac{\operatorname{ord}(\mathcal{G}) \operatorname{ord}(\mathcal{M}(\mathcal{G}))}{\operatorname{ord}\left(\mathcal{G}_{1}\right) \operatorname{ord}(\mathcal{M}(\mathcal{G}))}=\operatorname{ord}\left(\mathcal{G} / \mathcal{G}_{1}\right),
\end{aligned}
$$

and we may now apply Theorem 4.3(2) as well as part (b) of its proof to obtain our corollary.

We are now in a position to describe the structure of the tame infinitesimal groups of height $\geq 2$. For $n \geq 0$, we let $\psi_{s}^{n}: \mathfrak{s l}(2) \rightarrow \mathfrak{n}_{n}$ be the $p$-semilinear map with values in the the $n$-dimensional nil-cyclic restricted Lie algebra $\mathfrak{n}_{n}:=\bigoplus_{i=0}^{n-1} k v_{0}^{[p]^{i}}$, given by

$$
\psi_{n}^{s}(e)=0=\psi_{n}^{s}(f) ; \quad \psi_{n}^{s}(h)=v_{0}
$$

relative to the standard basis $\{e, f, h\}$ of $\mathfrak{s l}(2)$. Denote by $\mathfrak{s l}(2)_{n}:=\mathfrak{s l}(2)_{\psi_{n}^{s}}$ the corresponding central extension of $\mathfrak{s l}(2)$ with kernel $\mathfrak{n}_{n}$, and by $\mathrm{SL}(2)_{1}^{n}$ the infinitesimal group of height 1 associated to $\mathfrak{s l}(2)_{n}$. (In view of [18, (6.1)], the isomorphism class of SL $(2)_{n}$ does not depend on the choice of the basis of $\mathfrak{n}_{n}$.) Given $r \geq 1$, the $r$-th Frobenius kernel $T_{r}$ of the standard torus $T$ of $\mathfrak{s l}(2)$ acts on $\mathfrak{s l}(2)_{1}^{n}$ via conjugation, and we can form the semidirect product $\mathrm{SL}(2)_{1}^{n} \rtimes T_{r}$. Denoting the multiplicative center of this group by $\mathcal{M}(r, n)$, we define (see [18, Section 6])

$$
\mathcal{Q}_{[r, n]}:=\left(\mathrm{SL}(2)_{1}^{n} \rtimes T_{r}\right) / \mathcal{M}(r, n) .
$$

Note that $\mathcal{Q}_{[r, 0]} \cong \mathcal{Q}_{[r]}$ for every $r \geq 1$. 
Theorem 4.5. Let $\mathcal{G}$ be an infinitesimal group with $\operatorname{ht}(\mathcal{G} / \mathcal{M}(\mathcal{G})) \geq 2$. Then the following statements are equivalent:

(1) The principal block $\mathcal{B}_{0}(\mathcal{G})$ is tame.

(2) $\mathcal{G} / \mathcal{M}(\mathcal{G}) \cong \mathcal{Q}_{[r, n]}$ for some $r \geq 2, n \geq 0$.

(3) $H(\mathcal{G})$ is representation-infinite, special biserial.

(4) $H(\mathcal{G})$ is tame.

Proof. (1) $\Rightarrow(2)$ We have $\mathcal{B}_{0}(\mathcal{G}) \cong \mathcal{B}_{0}(\mathcal{G} / \mathcal{M}(\mathcal{G}))$ 20, (1.1)]. We proved in [18, (6.4)] that the principal block $\mathcal{B}_{0}(\mathcal{G} / \mathcal{M}(\mathcal{G}))$ is tame if and only if $\mathcal{G} / \mathcal{M}(\mathcal{G}) \cong \mathcal{Q}_{[r, n]}$ for $r=\operatorname{ht}(\mathcal{G} / \mathcal{M}(\mathcal{G}))$ and $n=\ell(\operatorname{Cent}(\mathcal{G}) / \mathcal{M}(\mathcal{G}))$.

$(2) \Rightarrow(3)$ This follows from [18, (6.4)], 20, (1.1)], and Theorem 4.3.

$(3) \Rightarrow(4)$ This follows from [43, (2.4)].

$(4) \Rightarrow(1)$ Since $H(\mathcal{G})$ is tame, the principal block $\mathcal{B}_{0}(\mathcal{G})$ is tame or representationfinite. In the latter case, [19, (2.7)] implies that $H(\mathcal{G})$ has finite representation type, a contradiction. Consequently, $\mathcal{B}_{0}(\mathcal{G})$ is tame.

Corollary 4.6. Let $\mathcal{G}$ be an infinitesimal group. Then the following statements are equivalent:

(1) $H(\mathcal{G})$ is tame.

(2) $H(\mathcal{G})$ is representation-infinite, special biserial.

Proof. In view of $[43,(2.4)]$ and Theorem 4.5, we only have to verify $(1) \Rightarrow(2)$ in case $\operatorname{ht}(\mathcal{G} / \mathcal{M}(\mathcal{G}))=1$. By the above arguments, $\mathcal{B}_{0}(\mathcal{G})$ is tame and Lemma 3.6 yields $\mathcal{G}=\mathcal{G}_{1} \mathcal{M}(\mathcal{G})$. Owing to Lemma 4.1 the algebra $H\left(\mathcal{G}_{1}\right) \cong U_{0}(\mathfrak{g})$ is tame. Hence $\mathcal{B}_{0}\left(\mathcal{G}_{1}\right)$ is tame and [17, (8.10)] shows that $H\left(\mathcal{G}_{1}\right)$ is representation-infinite, special biserial. Another application of Theorem 4.3 then yields our assertion.

Remarks. (1) According to [19, (2.7)], the algebra of measures $H(\mathcal{G})$ of an infinitesimal group $\mathcal{G}$ is representation-finite if and only if $H(\mathcal{G})$ is a Nakayama (hence special biserial) algebra.

(2) In view of [17, (8.10)] the implication $(4) \Rightarrow(1)$ in Theorem 4.5 fails for wild infinitesimal groups.

\section{The stable Auslander-Reiten quiver}

In this section we are concerned with the Auslander-Reiten theory of tame infinitesimal groups of characteristic $p \geq 3$.

Given a self-injective algebra $\Lambda$, we denote by $\Gamma_{s}(\Lambda)$ its stable Auslander-Reiten quiver. By definition, $\Gamma_{s}(\Lambda)$ is a directed graph, whose vertices are the isoclasses $[M]$ of the finite dimensional non-projective indecomposable $\Lambda$-modules $M$, and whose arrows are given by the irreducible morphisms. The graph $\Gamma_{s}(\Lambda)$ also possesses an automorphism $\tau$, the so-called Auslander-Reiten translation that reflects homological properties of $\Lambda$-modules. In fact, if $\Lambda$ is symmetric, then $\tau([M])=\left[\Omega_{\Lambda}^{2}(M)\right]$ is computable from the square of the Heller operator $\Omega_{\Lambda}$ for every $[M] \in \Gamma_{s}(\Lambda)$. We refer the reader to [3] and [4 for further details. We also note that if $\mathcal{G}$ is an infinitesimal group of characteristic $p \geq 3$ with tame principal block, then $H(\mathcal{G})$ is a symmetric algebra [18, (7.2)].

The representation theory of infinitesimal group schemes affords an analogue of Webb's Theorem [45, Theorem A] for finite groups: the tree class of a connected component of the stable Auslander-Reiten quiver $\Gamma_{s}(\mathcal{G})=\Gamma_{s}(H(\mathcal{G}))$ of an infinitesimal group $\mathcal{G}$ is either a finite Dynkin diagram, an infinite Dynkin diagram, or 
a Euclidean diagram (see [14, (1.3)]). The shapes of the connected components of the stable Auslander-Reiten quivers of the tame infinitesimal groups of height $\leq 1$ and the tame semisimple infinitesimal groups of characteristic $p \geq 3$ were described in [17, (7.2), (8.10)] and [20, (5.6)], respectively. Adopting the notation established in [12], we let $\tilde{\mathbb{A}}_{m, n}$ be the quiver with underlying vertex set $\mathbb{Z} /(m+n)$, clockwise oriented arrows $i \mapsto i+1,1 \leq i \leq m$, and counter-clockwise oriented arrows $i \mapsto i-1,1 \leq i \leq n$. Consequently, the tree class of $\mathbb{Z}\left[\tilde{\mathbb{A}}_{m, n}\right]$ is $A_{\infty}^{\infty}$ for $m n \geq 2$ and the Kronecker quiver $\tilde{\mathbb{A}}_{12}$, otherwise.

We now have the following general result.

Theorem 5.1. Let $\mathcal{G}$ be an infinitesimal group of characteristic $p \geq 3$ with tame principal block, $r:=\operatorname{ht}(\mathcal{G} / \mathcal{M}(\mathcal{G}))$ and $n:=\ell(\operatorname{Cent}(\mathcal{G}) / \mathcal{M}(\mathcal{G}))$. Let $\mathcal{B}$ be a tame block of $H(\mathcal{G})$. Then

(1) For $n=0$ and $r=1, \Gamma_{s}(\mathcal{B})$ consists of 2 components of Euclidean type $\mathbb{Z}\left[\tilde{\mathbb{A}}_{12}\right]$, and infinitely many components of type $\mathbb{Z}\left[\mathbb{A}_{\infty}\right] /(\tau)$.

(2) For $n=0$ and $r \geq 2, \Gamma_{s}(\mathcal{B})$ consists of 2 components of Euclidean type $\mathbb{Z}\left[\tilde{\mathbb{A}}_{p^{r-1}, p^{r-1}}\right], 4$ components of type $\mathbb{Z}\left[\mathbb{A}_{\infty}\right] /\left(\tau^{p^{r-1}}\right)$, and infinitely many components of type $\mathbb{Z}\left[\mathbb{A}_{\infty}\right] /(\tau)$.

(3) For $n \geq 1, \Gamma_{s}(\mathcal{B})$ consists of 4 components of type $\mathbb{Z}\left[\mathbb{A}_{\infty}\right] /\left(\tau^{p^{r-1}}\right)$, infinitely many components of type $\mathbb{Z}\left[\mathbb{A}_{\infty}\right] /(\tau)$, and infinitely many components of type $\mathbb{Z}\left[\mathbb{A}_{\infty}^{\infty}\right]$

Proof. We know from [17, (8.10)] and Theorem 4.3 that the block $\mathcal{B}$ is Morita equivalent to the symmetric special biserial algebra $\mathcal{N}^{2}(r, n)=k\left[\Delta_{r}\right] / J_{r, n}$. For $n=0, \mathcal{N}^{2}(r, n)$ is isomorphic to the trivial extension $H \ltimes D(H)$ of a radical square zero hereditary algebra $H$ of Euclidean type $\tilde{\mathbb{A}}_{2 p^{r-1}-1}\left(\right.$ with $\left.\tilde{\mathbb{A}}_{1}=\tilde{\mathbb{A}}_{12}\right)$ by its minimal injective cogenerator $D(H):=\operatorname{Hom}_{k}(H, k)$. Thus, statements (1) and (2) follow from [36, (3.6)] and [42]. Moreover, for $n \geq 1$ and $r=1$, statement (3) follows from [17, (7.2)].

Assume $n \geq 1$ and $r \geq 2$. Since $n \geq 1$, the bound quiver $\left(\Delta_{r}, J_{r, n}\right)$ has infinitely many primitive walks (primitive $V$-sequences in the sense of [43, (2.2)]), for example

$$
\alpha_{1}^{-1} \beta_{2}^{-1}\left(\alpha_{0} \beta_{1}\right)^{2}\left(\alpha_{1}^{-1} \beta_{2}^{-1} \alpha_{0} \beta_{1}\right)^{m} \quad, m \geq 1,
$$

and hence applying [12, (2.2)] we infer that $\Gamma_{s}\left(\mathcal{N}^{2}(r, n)\right)$ is a disjoint union of a finite number of components of type $\mathbb{Z}\left[\mathbb{A}_{\infty}\right] /\left(\tau^{m}\right)$ with $m \geq 2$, infinitely many components of type $\mathbb{Z}\left[\mathbb{A}_{\infty}\right] /(\tau)$, and infinitely many components of type $\mathbb{Z}\left[\mathbb{A}_{\infty}^{\infty}\right]$. Further, the mouth of every stable tube $\mathbb{Z}\left[\mathbb{A}_{\infty}\right] /\left(\tau^{m}\right)$ of rank $m \geq 2$ consists of the isoclasses of string modules (in the sense of [5]), whose Auslander-Reiten sequences have indecomposable middle terms. Moreover, by [5] (see also [11, (II.6.2), (IV.4.2), (IV.4.3)]) there is a bijection between the Auslander-Reiten sequences of string modules over $\mathcal{N}^{2}(r, n)$ with indecomposable middle terms and the arrows of the Gabriel quiver $\Delta_{r}$ of $\mathcal{N}^{2}(r, n)$, so that we have $4 p^{r-1}$ of them. In fact, the ends of such Auslander-Reiten sequences are the maximal uniserial submodules of the radicals of indecomposable projective $\mathcal{N}^{2}(r, n)$-modules. We will now describe these modules and their associated Auslander-Reiten sequences.

For a string $L$ of the bound quiver $\left(\Delta_{r}, J_{r, n}\right)$ we denote by $M(L)$ the associated (indecomposable) string $\mathcal{N}^{2}(r, n)$-module. For each vertex $i \in \mathbb{Z} /\left(2 p^{r-1}\right)$ of $\Delta_{r}$, we have two canonical strings

$$
u_{i}:=\left(\alpha_{i} \beta_{i+1}\right)^{p^{n}-1} \alpha_{i} \quad \text { and } \quad v_{i}:=\left(\beta_{i} \alpha_{i-1}\right)^{p^{n}-1} \beta_{i}
$$


such that $M\left(u_{i}\right)$ and $M\left(v_{i}\right)$ are uniserial $\mathcal{N}^{2}(r, n)$-modules with

$$
M\left(u_{i}\right)+M\left(v_{i+2}\right)=\operatorname{Rad} P(i+1) \quad \text { and } \quad M\left(u_{i}\right) \cap M\left(v_{i+2}\right)=\operatorname{Soc} P(i+1),
$$

where $P(j)$ denotes the indecomposable projective module associated to a vertex $j \in \mathbb{Z} /\left(2 p^{r-1}\right)$. Also consider the following strings:

$$
w_{i}:=u_{i} \beta_{i}^{-1} u_{i-2} \quad \text { and } \quad z_{i}:=v_{i} \alpha_{i}^{-1} v_{i+2}, \quad i \in \mathbb{Z} /\left(2 p^{r-1}\right),
$$

and the associated string $\mathcal{N}^{2}(r, n)$-modules $M\left(w_{i}\right)$ and $M\left(z_{i}\right)$. Then we have the following canonical Auslander-Reiten sequences of string $\mathcal{N}^{2}(r, n)$-modules:

$$
(0) \longrightarrow M\left(u_{i-2}\right) \longrightarrow M\left(w_{i}\right) \longrightarrow M\left(u_{i}\right) \longrightarrow(0)
$$

and

$$
(0) \longrightarrow M\left(v_{i+2}\right) \longrightarrow M\left(z_{i}\right) \longrightarrow M\left(v_{i}\right) \longrightarrow(0)
$$

for all $i \in \mathbb{Z} /\left(2 p^{r-1}\right)$. Therefore, we have 4 stable tubes of rank $p^{r-1}$ in $\Gamma_{s}\left(\mathcal{N}^{2}(r, n)\right)$, namely:

(a) $\mathcal{T}_{1}$, whose mouth consists of the isoclasses of the string modules $M\left(u_{0}\right), M\left(u_{2}\right), \ldots, M\left(u_{2 p^{r-1}-4}\right), M\left(u_{2 p^{r-1}-2}\right)$

(b) $\mathcal{T}_{2}$, whose mouth consists of the isoclasses of the string modules $M\left(u_{1}\right), M\left(u_{3}\right), \ldots, M\left(u_{2 p^{r-1}-3}\right), M\left(u_{2 p^{r-1}-1}\right)$

(c) $\mathcal{T}_{3}$, whose mouth consists of the isoclasses of the string modules $M\left(v_{2 p^{r-1}-2}\right), M\left(v_{2 p^{r-1}-4}\right), \ldots, M\left(v_{2}\right), M\left(v_{0}\right)$

(d) $\mathcal{T}_{4}$, whose mouth consists of the isoclasses of the string modules $M\left(v_{2 p^{r-1}-1}\right), M\left(v_{2 p^{r-1}-3}\right), \ldots, M\left(v_{3}\right), M\left(v_{1}\right)$.

Therefore, $\mathcal{T}_{1}, \mathcal{T}_{2}, \mathcal{T}_{3}$ and $\mathcal{T}_{4}$ are the unique stable tubes of $\Gamma_{s}\left(\mathcal{N}^{2}(r, n)\right)$ of ranks $\geq 2$, and this completes the proof of $(3)$.

Theorem 5.1 elicits the significance of the invariant $r:=\operatorname{ht}(\mathcal{G} / \mathcal{M}(\mathcal{G}))$ for the representation theory of $\mathcal{G}$. Our next result, which elaborates on [15, (4.4)], underscores this fact from a different perspective. Letting $\tau$ denote the Auslander-Reiten translation of $\Gamma_{s}(\mathcal{G})$, we recall that a non-projective indecomposable $\mathcal{G}$-module $M$ is referred to as $\tau$-periodic if $\tau^{n}([M])=[M]$ for some $n \geq 1$. By general theory [3, (IV.3.7)], the Auslander-Reiten translation is computable from the Heller operator $\Omega$ and the Nakayama functor $\nu$ of $\bmod \mathcal{G}$ via the formula

$$
\tau=\Omega^{2} \circ \nu=\nu \circ \Omega^{2} .
$$

Since the Nakayama functor of $H(\mathcal{G})$ has finite order (cf. [29, (I.8.12)]), the notions of $\tau$-periodicity and $\Omega$-periodicity coincide.

Proposition 5.2. Let $M$ be a $\tau$-periodic module of the infinitesimal $k$-group $\mathcal{G}$ and put $r:=\operatorname{ht}(\mathcal{G} / \mathcal{M}(\mathcal{G}))$.

(1) We have $\tau^{p^{r}}([M])=[M]$.

(2) If $H(\mathcal{G})$ is symmetric, then we have $\tau^{p^{r-1}}([M])=[M]$.

Proof. Since $\mathcal{M}(\mathcal{G})$ is multiplicative, the Hochschild-Serre spectral sequence

$$
H^{n}\left(\mathcal{G} / \mathcal{M}(\mathcal{G}), H^{m}(\mathcal{M}(\mathcal{G}), k)\right) \Rightarrow H^{n+m}(\mathcal{G}, k)
$$

collapses to isomorphisms $\gamma_{n}: H^{n}(\mathcal{G} / \mathcal{M}(\mathcal{G}), k) \stackrel{\sim}{\longrightarrow} H^{n}(\mathcal{G}, k)$. The isomorphisms $\gamma_{n}$ are the edge homomorphisms $E_{2}^{n, 0} \longrightarrow H^{n}(\mathcal{G}, k)$ of the spectral sequence, and 
are thus induced from the canonical inflation map $H^{*}(\mathcal{G} / \mathcal{M}(\mathcal{G}), k) \longrightarrow H^{*}(\mathcal{G}, k)$ (cf. [46, (5.8.3)]). As a result, the commutative $k$-algebras

$$
H^{\mathrm{ev}}(\mathcal{G}, k):=\bigoplus_{n \in \mathbb{N}_{0}} H^{2 n}(\mathcal{G}, K)
$$

and $H^{\mathrm{ev}}(\mathcal{G} / \mathcal{M}(\mathcal{G}), k)$ are isomorphic. By the Friedlander-Suslin Theorem 22, (1.5)], the algebra $H^{\mathrm{ev}}(\mathcal{G}, k)$ thus has a system of homogeneous parameters of degrees $2 p^{i}, 0 \leq i \leq r-1$.

Let $\zeta \in X(\mathcal{G})$ be the modular function of the Hopf algebra $H(\mathcal{G})$. Since $\mathcal{M}(\mathcal{G})$ lies centrally in $\mathcal{G}, \zeta$ annihilates the augmentation ideal of $H(\mathcal{M}(\mathcal{G})$ ) (cf. [29, (I.8.8)]). Consequently, $\zeta$ belongs to the subgroup $X(\mathcal{G} / \mathcal{M}(\mathcal{G}))$ and thus has order a divisior of $p^{r}$. The arguments of [15, (4.4)] now yield the assertions.

\section{INFINITESIMAL GROUPS OF POLYNOMIAL GROWTH}

Let $\Lambda$ be a tame algebra. For each $d \in \mathbb{N}$, we let $\mu_{\Lambda}(d)$ be the minimum number of $(\Lambda, k[X])$-bimodules $M_{1}, \ldots, M_{\mu_{\Lambda}(d)}$ giving rise to the one-parameter families of indecomposable $d$-dimensional $\Lambda$-modules. The algebra $\Lambda$ is said to have polynomial growth if there exists a natural number $m$ such that $\mu_{\Lambda}(d) \leq d^{m}$ for every $d \geq 1$ (see [39]). Moreover, we refer to $\Lambda$ as being domestic if there exists a natural number $m$ such that $\mu_{\Lambda}(d) \leq m$ for every $d \geq 1$ (see [8, (5.7)]). It is well-known that an algebra $\Lambda$ is of polynomial growth (respectively, domestic) if and only if its basic algebra enjoys this property.

Theorem 6.1. Let $\mathcal{G}$ be an infinitesimal group of characteristic $p \geq 3$. Then the following statements are equivalent:

(1) $\mathcal{B}_{0}(\mathcal{G})$ is of polynomial growth.

(2) $\mathcal{G} / \mathcal{M}(\mathcal{G}) \cong \mathcal{Q}_{[r]}$ for some $r \geq 1$.

(3) $H(\mathcal{G})$ is domestic with $\mu_{H(\mathcal{G})}=2$.

(4) $H(\mathcal{G})$ is tame and $\Gamma_{s}(\mathcal{G})$ admits a connected component of Euclidean type.

Proof. $(1) \Rightarrow(2)$. Suppose that $\mathcal{B}_{0}(\mathcal{G})$ is of polynomial growth. Then $\mathcal{B}_{0}(\mathcal{G})$ is tame and hence is Morita equivalent to $\mathcal{N}^{2}(r, n)$ for $r=h t(\mathcal{G} / \mathcal{M}(\mathcal{G}))$ and $n=$ $\ell(\operatorname{Cent}(\mathcal{G}) / \mathcal{M}(\mathcal{G}))$ by [18, (7.1)]. Since $\mathcal{N}^{2}(r, n)$ is of polynomial growth we infer from [12, (2.1)] that its bound quiver $\left(\Delta_{r}, J_{r, n}\right)$ admits only finitely many primitive walks, and consequently $n=0$ (see the proof of Theorem 5.1(3)). On the other hand, we know from Theorem 1.1 that $\mathcal{G} / \operatorname{Cent}(\mathcal{G}) \cong \mathcal{Q}_{[r]}$ and $\operatorname{Cent}(\mathcal{G}) \cong \mathcal{M}(\mathcal{G}) \times$ $\left(\mathcal{W}_{n}\right)_{1}$, where $\left(\mathcal{W}_{n}\right)_{1}$ is the first Frobenius kernel of the group $\mathcal{W}_{n}$ of Witt vectors of length $n$. Hence $n=0$ implies $\operatorname{Cent}(\mathcal{G}) \cong \mathcal{M}(\mathcal{G})$, and (2) holds.

$(2) \Rightarrow(3)$. Assume $\mathcal{G} / \mathcal{M}(\mathcal{G}) \cong \mathcal{Q}_{[r]}$ for some $r \geq 1$. Clearly then $r=$ $\operatorname{ht}(\mathcal{G} / \mathcal{M}(\mathcal{G}))$. Moreover $\mathcal{B}_{0}(\mathcal{G}) \cong \mathcal{B}_{0}(\mathcal{G} / \mathcal{M}(\mathcal{G}))$ is Morita equivalent to $\mathcal{N}^{2}(r, 0)$, and consequently is tame. For $r \geq 2$, Theorem 4.3 implies that every block of $H(\mathcal{G})$ is Morita equivalent to $\mathcal{N}^{2}(r, 0)$ or to $k[X] /(X)=k$. For $r=1$, we first apply Lemma 3.6 to see that $\mathcal{G}=\mathcal{G}_{1} \mathcal{M}(\mathcal{G})$. In view of Lemma 4.1 it therefore suffices to study the blocks of the algebra $H\left(\mathcal{G}_{1}\right) \cong U_{0}(\mathfrak{g})$. As usual, $\mathfrak{g}:=\operatorname{Lie}(\mathcal{G})$ is the Lie algebra of $\mathcal{G}$.

Since $\mathcal{Q}_{[r]}$ is semisimple, our present assumption implies $\mathcal{M}(\mathcal{G})=\operatorname{Cent}(\mathcal{G})$. The presentation $\mathcal{G}=\mathcal{G}_{1} \mathcal{M}(\mathcal{G})$ yields $\operatorname{Cent}\left(\mathcal{G}_{1}\right) \subset \operatorname{Cent}(\mathcal{G})$, so that the center of $\mathfrak{g}$ is a torus. The arguments of the proof of [17, (8.10)] now show that every block of $H(\mathcal{G})$ is Morita equivalent to $\mathcal{N}^{2}(1,0), k[X] /(X)$, or $k[X] /\left(X^{2}\right)$. In summation, the 
blocks of $H(\mathcal{G})$ are either Nakayama algebras, or Morita equivalent to $\mathcal{N}^{2}(r, 0)$. As noted in the proof of Theorem 5.1, the algebra $\mathcal{N}^{2}(r, 0)$ is isomorphic to the trivial extension $H \ltimes D(H)$ of the radical square zero hereditary algebra $H$ of Euclidean type $\tilde{\mathbb{A}}_{2 p^{r-1}-1}$ (with $\tilde{\mathbb{A}}_{1}=\tilde{\mathbb{A}}_{12}$ ). By results of Tachikawa [42], the latter algebra is 2-parametric, so that $H(\mathcal{G})$ is domestic with $\mu_{H(\mathcal{G})}=2$.

$(3) \Rightarrow(4)$. This is a direct consequence of Corollary [4.6 and [12, (2.1)].

$(4) \Rightarrow(1)$. This is a direct consequence of Corollary 4.6 and [12, (2.1), (2.2)].

\section{The Krull-Gabriel dimension of $H(\mathcal{G})$}

Given a $k$-algebra $\Lambda$, we denote by $\bmod \Lambda$ and $\mathcal{C}(\Lambda)$ the categories of finite dimensional left $\Lambda$-modules and finitely presented covariant functors from $\bmod \Lambda$ to $\bmod k$, respectively. We will write $\mathcal{A} / \mathcal{S}$ for the abelian quotient of an abelian category $\mathcal{A}$ by a Serre subcategory $\mathcal{S}$ (cf. [27, (1.11)] and [38, (I.2)]). We put $\mathcal{C}_{-1}:=0$, and define $\mathcal{C}_{n}$ to be the category of all functors $F \in \mathcal{C}$ that are sent to an object of finite length by the canonical quotient functor $\mathcal{C} \rightarrow \mathcal{C} / \mathcal{C}_{n-1}$. Following 23] we define the Krull-Gabriel dimension $\operatorname{KG}(\Lambda) \in \mathbb{N} \cup\{\infty\}$ via

$$
\operatorname{KG}(\Lambda):=\min \left\{n \in \mathbb{N} \cup\{\infty\} ; \mathcal{C}_{n}=\mathcal{C}\right\} .
$$

It was shown in [1, (3.14)] that $\operatorname{KG}(\Lambda)=0$ if and only if $\Lambda$ is of finite representation type.

A commutative unipotent infinitesimal $k$-group $\mathcal{U}$ is called $V$-uniserial if the Verschiebung $V_{\mathcal{U}}: \mathcal{U}^{(p)} \longrightarrow \mathcal{U}$ (cf. [9, (IV,§3,no.4),(II,§7,no.1)]) induces an exact sequence

$$
\mathcal{U}^{(p)} \stackrel{V_{\mathcal{u}}}{\longrightarrow} \mathcal{U} \longrightarrow \alpha_{p} \longrightarrow e_{k} .
$$

Here $\alpha_{p}:=\operatorname{Spec}_{k}\left(k[T] /\left(T^{p}\right)\right)$ denotes the first Frobenius kernel of the additive group.

Theorem 7.1. Let $\mathcal{G}$ be an infinitesimal group of characteristic $p \geq 3$. Then the following statements are equivalent:

(1) $\operatorname{KG}\left(\mathcal{B}_{0}(\mathcal{G})\right)<\infty$

(2) $\mathcal{G} / \mathcal{M}(\mathcal{G}) \cong \mathcal{Q}_{[r]}$ for some $r \geq 1$, or $\mathcal{G} / \mathcal{M}(\mathcal{G}) \cong \mathcal{U} \rtimes \mu_{p^{m}}$ for a multiplicative group $\mu_{p^{m}}$ and a $V$-uniserial normal subgroup $\mathcal{U}$.

(3) $\operatorname{KG}(H(\mathcal{G})) \in\{0,2\}$.

Remark. Owing to [16, (4.2)] the structure of the groups $\mathcal{G} / \mathcal{M}(\mathcal{G}) \cong \mathcal{U} \rtimes \mu_{p^{m}}$ is well understood (see also 31] for uniserial groups).

Proof. (1) $\Rightarrow(2)$. Suppose $\mathcal{B}_{0}(\mathcal{G})$ has finite Krull-Gabriel dimension. Owing to 24] this implies that $\mathcal{B}_{0}(\mathcal{G})$ is of finite or tame representation type. In the former case, the classification of representation-finite infinitesimal groups (cf. [19, (2.7)]) shows that $\mathcal{G} / \mathcal{M}(\mathcal{G})$ is a semidirect product of a multiplicative group $\mu_{p^{m}}$ and a $V$-uniserial normal subgroup $\mathcal{U}$. If $\mathcal{B}_{0}(\mathcal{G})$ is tame, then [20, (1.1)] and [18, Thm.B] imply that $\mathcal{B}_{0}(\mathcal{G}) \cong \mathcal{B}_{0}(\mathcal{G} / \mathcal{M}(\mathcal{G}))$ is Morita equivalent to a double Nakayama algebra $\mathcal{N}^{2}(r, n)$ for some $r \geq 1$ and $n \geq 0$. Suppose $n \geq 1$. Then the string algebra $\mathcal{B}_{0}(\mathcal{G}) / \operatorname{Soc} \mathcal{B}_{0}(\mathcal{G})$ is non-domestic (cf. [12, (2.1)]) and in view of [37, Prop. 2] has infinite Krull-Gabriel dimension. Thus, $\operatorname{KG}\left(\mathcal{B}_{0}(\mathcal{G})\right)=\infty$, and we have reached a contradiction. Therefore, $n=0$ and $\mathcal{B}_{0}(\mathcal{G})$ is a domestic algebra. Now applying Theorem 6.1 we obtain $\mathcal{G} / \mathcal{M}(\mathcal{G}) \cong \mathcal{Q}_{[r]}$ for some $r \geq 1$. 
$(2) \Rightarrow(3)$. If $\mathcal{G}$ is given as in (2), then Theorem 6.1] and [19, (2.7)] show that $H(\mathcal{G})$ is of tame or finite representation type. In the latter case, [1, (3.14)] yields $\operatorname{KG}(H(\mathcal{G}))=0$. Alternatively, we have $\mathcal{G} / \mathcal{M}(\mathcal{G}) \cong \mathcal{Q}_{[r]}$, and then the proof of Theorem 6.1 shows that $H(\mathcal{G})$ is a domestic algebra whose tame blocks are Morita equivalent to $\mathcal{N}^{2}(r, 0)$. Since $\mathcal{N}^{2}(r, 0)$ is the trivial extension $H \ltimes D(H)$ of a hereditary algebra $H$ of Euclidean type $\tilde{\mathbb{A}}_{2 p^{r-1}-1}$, an application of [24, (3.9)] and [42] gives $\operatorname{KG}\left(\mathcal{N}^{2}(r, 0)\right)=2$. Since all other blocks are representation-finite algebras, $H(\mathcal{G})$ has Krull-Gabriel dimension 2.

(3) $\Rightarrow(1)$. Trivial.

\section{ACKNOWLEDGEMENTS}

Major parts of this paper were written during the first author's stay at Nicolaus Copernicus University in May of 2003. He gratefully acknowledges the support by the Foundation for Polish Science and thanks the Faculty of Mathematics and Computer Science for its hospitality.

\section{REFERENCES}

[1] M. Auslander. A functorial approach to representation theory. In: Representations of Algebras. Lecture Notes in Mathematics 944 (1980), 105-179. MR0672116 (83m:16027)

[2] M. Auslander, I. Reiten, and S. Smalø. Galois actions on rings and finite Galois coverings. Math. Scand. 65 (1989), 5-32. MR1051819 (91e:16047)

[3] M. Auslander, I. Reiten, and S. Smalø. Representation Theory of Artin Algebras. Cambridge Studies in Advanced Mathematics 36. Cambridge University Press, 1995. MR 1314422 (96c:16015)

[4] D. Benson. Representations and Cohomology, I. Cambridge Studies in Advanced Mathematics 30. Cambridge University Press, 1991. MR1110581 (92m:20005)

[5] M. Butler and C. Ringel. Auslander-Reiten sequences with few middle terms and applications to string algebras. Comm. Algebra 15 (1987), 145-179. MR0876976 (88a:16055)

[6] H. Cartan and S. Eilenberg. Homological Algebra. Princeton Mathematical Series 19. Princeton University Press, Princeton, 1956. MR0077480 (17:1040e)

[7] W. Crawley-Boevey. On tame algebras and bocses. Proc. London Math. Soc. 56 (1988), 451483. MR0931510 (89c:16028)

[8] W. Crawley-Boevey. Tame algebras and generic modules. Proc. London Math. Soc. 63 (1991), 241-265. MR1114510 (92m:16019)

[9] M. Demazure and P. Gabriel. Groupes Algébriques. Masson, Paris/North Holland, Amsterdam, 1970. MR0302656 (46:1800)

[10] Yu. Drozd. Tame and wild matrix problems, in: Representation Theory II. Lecture Notes in Mathematics 832 (1980), 242-258. MR0607157 (83b:16024)

[11] K. Erdmann. Blocks of Tame Representation Type and Related Algebras. Lecture Notes in Mathematics 1428. Springer-Verlag, 1990. MR.1064107 (91c:20016)

[12] K. Erdmann and A. Skowroński. On Auslander-Reiten components and self-injective biserial algebras. Trans. Amer. Math. Soc. 330 (1992), 165-189. MR.1144759 (93b:16022)

[13] R. Farnsteiner. Periodicity and representation type of modular Lie algebras. J. reine angew. Math. 464 (1995), 47-65. MR.1340334 (97a:17019)

[14] R. Farnsteiner. On the Auslander-Reiten quiver of an infinitesimal group. Nagoya Math. J. 160 (2000), 103-121. MR.1804139 (2001k:16027)

[15] R. Farnsteiner. Auslander-Reiten components for $G_{1} T$-modules. J. Algebra Appl. 4 (2005), 739-759. MR2192153(2006i:16026)

[16] R. Farnsteiner, G. Röhrle, and D. Voigt. Infinitesimal unipotent group schemes of complexity 1. Colloq. Math. 89 (2001), 179-192. MR1854701(2002m:14039)

[17] R. Farnsteiner and A. Skowroński. Classification of restricted Lie algebras with tame principal block. J. reine angew. Math. 546 (2002), 1-45. MR1900992 (2003c:17031) 
[18] R. Farnsteiner and A. Skowroński. The tame infinitesimal groups of odd characteristic. Adv. in Math. 205 (2006), 229-274. MR2254312 (2007d:14082)

[19] R. Farnsteiner and D. Voigt. On cocommutative Hopf algebras of finite representation type. Adv. in Math. 155 (2000), 1-22. MR1789845 (2001e:16070)

[20] R. Farnsteiner and D. Voigt. On infinitesimal groups of tame representation type. Math. Z. 244 (2003), 479-513. MR.1992021 (2004c:16063)

[21] E. Friedlander and B. Parshall. Modular representation theory of Lie algebras. Amer. J. Math. 110 (1988), 1055-1094. MR0970120 (89j:17015)

[22] E. Friedlander and A. Suslin. Cohomology of finite group schemes over a field. Invent. math. 127 (1997), 209-270. MR 1427618 (98h:14055a)

[23] W. Geigle. The Krull-Gabriel dimension of the representation theory of a tame hereditary algebra and applications to the structure of exact sequences. Manuscr. math 54 (1985), 83106. MR0808682 (87e:16061)

[24] W. Geigle. Krull dimension of Artin algebras. In: Representation Theory I. Lecture Notes in Mathematics 1177 (1984), 135-155. MR0842464 (87j:16008)

[25] I. Gel'fand and V. Ponomarev. Indecomposable representations of the Lorentz group. Usp. Math. Nauk 23 (1968), 1-58. MR0229751(37:5325)

[26] R. Gordon and E. Green. Graded Artin algebras. J. Algebra 76 (1982), 111-137. MR0659212 $(83 \mathrm{~m}: 16028 \mathrm{a})$

[27] A. Grothendieck. Sur quelques points d'algèbre homologique. Tôhoku Math. J. 9 (1957), 119221. MR0102537(21:1328)

[28] J. Jantzen. Über Darstellungen höherer Frobenius-Kerne halbeinfacher algebraischer Gruppen. Math. Z. 164 (1979), 271-292. MR0516611 (80f:20044)

[29] J. Jantzen. Representations of Algebraic Groups. Pure and Applied Mathematics 131. Academic Press, 1987. MR0899071 (89c:20001)

[30] J. Jantzen. Modular representations of reductive Lie algebras. J. Pure Appl. Algebra 152 (2000), 133-185. MR1783993 (2001j:17016)

[31] A. Koch. The Hopf algebra of a uniserial group. Pacific J. Math. 215 (2004), 347-356. MR2068786 (2005f:16060)

[32] S. Montgomery. Hopf Algebras and their Actions on Rings. CBMS 82. Amer. Math. Soc., 1993. MR1243637 (94i:16019)

[33] U. Oberst and H. Schneider. Über Untergruppen endlicher algebraischer Gruppen. Manuscripta math. 8 (1973), 217-241. MR0347838 (50:339)

[34] W. Pfautsch. Ext ${ }^{1}$ for the Frobenius kernels of $\mathrm{SL}_{2}$. Comm. Algebra 13 (1985), 169-179. MR0768093 (86d:20052)

[35] J. Rotman. An Introduction to Homological Algebra. Pure and Applied Mathematics 85. Academic Press, 1979. MR0538169 (80k:18001)

[36] C. Ringel. Tame Algebras and Integral Quadratic Forms. Lecture Notes in Mathematics 1099. Springer-Verlag, 1984. MR0774589 (87f:16027)

[37] J. Schröer. On the Krull-Gabriel dimension of an algebra. Math. Z. 223 (2000), 287-303. MR:1743438 (2000m:16024)

[38] J.-P. Serre. Classes des groupes abéliens et groupes d'homotopie. Ann. Math. 58 (1953), 258-284. MR0059548 (15:548c)

[39] A. Skowroński. Group algebras of polynomial growth. Manuscr. math. 59 (1987), 499-516. MR0916000 (88m:16035)

[40] A. Skowroński and J. Waschbüsch. Representation-finite biserial algebras. J. reine angew. Math. 345 (1983), 172-181. MR0717892 (85e:16051)

[41] H. Strade and R. Farnsteiner. Modular Lie Algebras and their Representations. Marcel Dekker Monographs 116. New York, 1988. MR0929682 (89h:17021)

[42] H. Tachikawa. Representations of trivial extensions of hereditary algebras. In: Representation theory II. Lecture Notes in Mathematics 832, 579-599. Springer Verlag, 1980. MR0607173 (82d:16029)

[43] B. Wald and J. Waschbüsch. Tame biserial algebras. J. Algebra 95 (1985), 480-500. MR.0801283 (87a:16056)

[44] W. Waterhouse. Introduction to Affine Group Schemes. Lecture Notes in Mathematics 66. Springer Verlag, 1979. MR0547117 (82e:14003) 
[45] P. Webb. The Auslander-Reiten quiver of a finite group. Math. Z. 179 (1982), 97-121. MR0643050 (83b:16029)

[46] C. Weibel. An Introduction to Homological Algebra. Cambridge Studies in Advanced Mathematics 38. Cambridge University Press, 1994. MR1269324 (95f:18001)

Fakultät für Mathematik, Universität Bielefeld, Postfach 1001 31, 33501 Bielefeld, GERMAny

E-mail address: rolf@mathematik.uni-bielefeld.de

Faculty of Mathematics and Computer Science, Nicolaus Copernicus University, Chopina 12/18, 87-100 Toruń, Poland

E-mail address: skowron@mat.uni.torun.pl 